\title{
Electron Emission from Carbon Nanotubes
}

\author{
by \\ Parham Yaghoobi \\ B.A.Sc. (Electrical Engineering), The University Of British Columbia, 2007 \\ A THESIS SUBMITTED IN PARTIAL FULFILLMENT OF \\ THE REQUIREMENTS FOR THE DEGREE OF \\ MASTER OF APPLIED SCIENCE \\ in \\ The Faculty of Graduate Studies \\ (Electrical and Computer Engineering) \\ THE UNIVERSITY OF BRITISH COLUMBIA \\ (Vancouver) \\ March, 2009 \\ (c) Parham Yaghoobi 2009
}




\section{Abstract}

In this thesis, I will provide an overview of the subject from various experimental and theoretical angles with an emphasis on the limited number of theoretical works that exist in calculating the emission current. I will then present a first-principles calculation of the emission current in a single-walled carbon nanotube using the non-equilibrium Green's function and Fisher-Lee's transmission formulation to describe electronic transport through the system. The simulation results reproduce the trends observed in experimental data closely and, in particular, the current saturation behavior observed in experiments and the deviation from the Fowler-Nordheim behavior. The proposed numerical approach is useful whenever a region of vacuum is present in the device Hamiltonian, which existing commercial transport solvers do not model. To better characterize nanotube electron sources, I have also done some preliminary experimental work, which has involved building equipments and developing recipes for growing carbon nanotubes. 


\section{Contents}

Abstract ....................... . . . . . . . .

Contents ................................ ii

List of Figures . . . . . . . . . . . . . . . . . . . . . vi

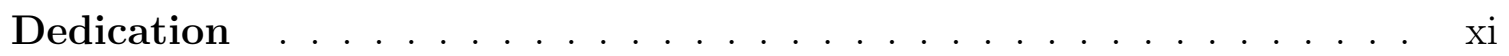

Statement of Co-Authorship ................ xii

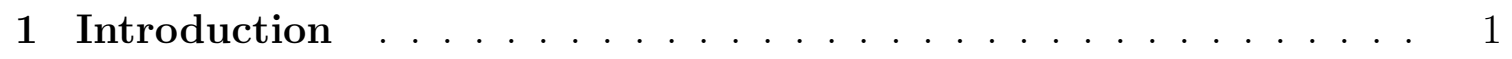

1.1 Motivation .......................... 1

1.2 Thesis Overview . . . . . . . . . . . . . . . . 3

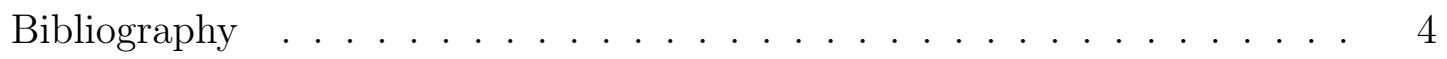

2 Electron Emission From Carbon Nanotubes . . . . . . . . . 5

2.1 Electron Emission Mechanisms . . . . . . . . . . . . 5

2.2 Carbon Nanotubes . . . . . . . . . . . . . . . . . 7 
2.3 Field-electron emission from individual nanotubes $\ldots \ldots \ldots \ldots$

2.3.1 Single-Walled Carbon Nanotubes . . . . . . . . . . . . . 9

2.3.2 Multi-Walled Carbon Nanotubes _... . . . . . . . . . . . 11

2.4 Field-Electron Emission from Collections of Nanotubes . . . . . . . 15

2.4.1 Single-Walled Carbon Nanotubes _. . . . . . . . . . . 15

2.4.2 Multi-Walled Carbon Nanotubes . . . . . . . . . . . 17

2.5 Other Traditional Emission Mechanisms . . . . . . . . . . . . . 19

2.6 Novel Electron Emission Mechanisms _ . . . . . . . . . . . . . . . . 21

2.7 Observation of Emission Sites $\ldots \ldots \ldots$

2.8 Modeling and Simulating Electron Emission From Carbon Nanotubes 26

2.9 Final Thoughts . . . . . . . . . . . . . . . . . . 29

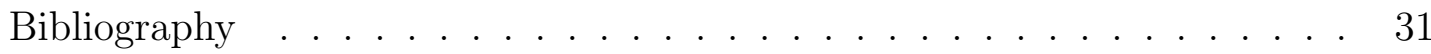

3 First-Principles Study of Quantum Tunneling from Nanostructures: Current in a Single-Walled Carbon Nanotube Electron Source . 37

3.1 Introduction $\ldots \ldots \ldots \ldots \ldots \ldots \ldots \ldots \ldots \ldots \ldots$

3.2 Methodology . . . . . . . . . . . . . . . . . . . . . 39

3.3 Results and Discussion . . . . . . . . . . . . . . . . 42

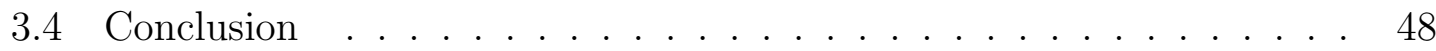

Bibliography . . . . . . . . . . . . . . . . . . 49 
4 Summary, Conclusions and Further Work . . . . . . . . . . 53

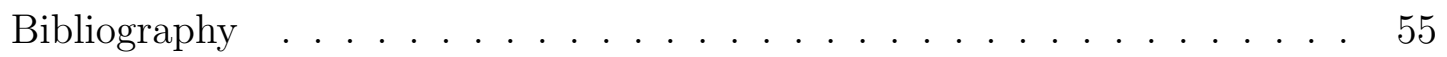

\section{Appendices}

A Experimental Setup _. . . . . . . . . . . . . . 56

A.1 Chemical Vapor Deposition system . . . . . . . . . . . . . 56

A.2 Scanning Electron Microscope . . . . . . . . . . . . . . . . 59

A.3 Ultra High Vacuum Chamber . . . . . . . . . . . . . . . 61

Bibliography ....................... 62 


\section{List of Figures}

1.1 A high-resolution image of a carbon nanotube suspended over the edge of a peeled metal electrode, obtained in an FEI Sirion scanning electron microscope. . . . . . . . . . . . . . . . . . . . 2

2.1 Various electron emission mechanisms: a) thermionic emission, b) field-emission, c) Schottky emission and d) photo-emission. . . . . . . 6

2.2 The hexagonal lattice of graphene and the unit vectors - rolling the graphene up along different directions will lead to nanotubes with various chiralities. . . . . . . . . . . . . . . . . . 7

2.3 The current saturation behavior of an individual SWNT - the dashed line is a FowlerNordheim equation fit to the low voltage data [10]. . . 10

2.4 (a) Schematic of a surface nanotube electron emitter. (b) Emission current vs. applied voltage potted on an FN scale [11] . . . . . . . . . 11

2.5 (a) Scanning electron micrograph of a nanotube of length $\mathrm{h}=1.4 \mathrm{um}$ and radius $\mathrm{r}=7.5 \mathrm{~nm}$ with a sharp anode positioned at a distance $\mathrm{d}$ $=2.65 \mathrm{um}$. (b) The corresponding I-V curve with a fit to the FN law in the dotted line. The FN plot is given in the inset [13] . . . . . . . 11 
2.6 (a) IV characteristics of a MWNT with amorphous carbon on tip before (Group B) and after (Group A) the heat treatment. For the heat treatment, the tungsten tip was heated to $700 \mathrm{~K}$ during the field emission at $800 \mathrm{~V}$. (b) FN plot of the data with straight line fits in

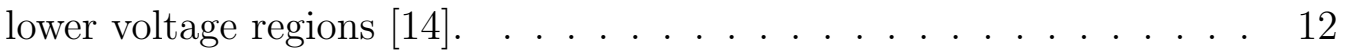

2.7 (a) $\mathrm{I}-\mathrm{V}$ curves of the field emission for different nanotube-anode distances d. (b) The corresponding F-N plots [15]. . . . . . . . . . . . . 13

2.8 (a) I-V curves of 5 different nanotubes. (b) The corresponding F-N plots $[16] \ldots \ldots \ldots \ldots \ldots \ldots$

2.9 Brightness $(\mathrm{Br})$ as a function of energy distribution $(\Delta E)$ measured for several CNTs at currents of $10500 \mathrm{nA}$ and temperatures of 600700 K. Calculated curve (dashed line). The data of the Schottky emitter and the tungsten cold field emission gun are also included [18]. . . . . 14

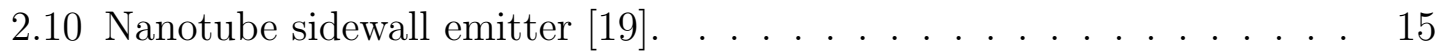

2.11 (a) Schematic geometry of a triode-type carbon nanotube-based field emission display. (b) Emission current of a triode type CNT-FED for various anode voltages. [20]. . . . . . . . . . . . . . . . . 16

2.12 (a) schematic diagram of flexible substrate and aligned carbon nanotubes. (b) Emission current density vs. electric field for the emitter. Inset: FN plot [21]. . . . . . . . . . . . . . . . . . . . . . . . . 16

2.13 Single I V characteristics of a typical SWNT film. Inset: the corresponding FN plot. Arrows (a) and (b) indicate the end of the low current regime and the onset of the saturation, respectively [6] . . . . 17

2.14 (a) I-V curve for field-emission from a MWNT matrix sample - it shows both logarithmic and linear current scales. (b) The corresponding FN plot [8]. 
2.15 (a) IV characteristics of CNT clusters synthesized by arc discharge with $150 \mathrm{~A}$ arc current and $20 \mathrm{~V}$ arc voltage, under 500 mbar (He); the inset shows the corresponding FN plot [24]. . . . . . . . . . . . 19

2.16 Bright spots at the nanotube tips as a result of beam-stimulated fieldemission. The wide semi-bright area on the right is the cathode electrode and the square is the nanotube growth catalyst island [32]. . . . 22

2.17 Bright emission spot at a nanotube tip emitting electrons, imaged in a transmission electron microscope, and structural damage to the nanotube tip [33]. . . . . . . . . . . . . . . . . . . . 2

2.18 Electron emission patterns from a SWNT in a field-emission microscope $[35] \ldots \ldots \ldots \ldots \ldots \ldots \ldots$

2.19 Deformed nanotube tip as a result of electron emission, seen in a transmission electron microscope [38]. . . . . . . . . . . . . . .

3.1 (A) Schematic representation of SWNT, consisting of the emitter grown on a cathode and biased with respect to an anode. (B) Enlarged view of the tip of the SWNT and vacuum. . . . . . . . . 39

3.2 The simulated device, which encapsulates both the SWNT and vacuum. It consists of 8 unit-cells of a $(5,5)$ SWNT capped with half a $C_{60}$ molecule. The other end of the nanotube is terminated with hydrogen to avoid dangling bonds. $\Sigma_{1}$ is calculated $5 \AA$ away from the hydrogen termination because the $\mathrm{H}-\mathrm{C}$ produces an undesired dipole at the end as the result of the difference in electronegativity. $\Sigma_{2}$ is calculated $10 \AA$ away from the tip of the structure since this is where the vacuum level has been found to be unperturbed by the molecule. 
3.3 Mulliken charge distribution along the SWNT at: (A) $0.1 \mathrm{VA}^{-1}$, (B) $0.6 \mathrm{~V}^{-1}$, and (C) $1.2 \mathrm{~V}^{-1}$. The lightest grey atoms illustrate negative charges of approximately 0.17 electrons and black atoms illustrate 0 charge. . . . . . . . . . . . . . . . . . . . . . . .

3.4 The I-V characteristics of the SWNT. The current has a pseudoexponential behavior in the turn-on $0.2-0.3 \mathrm{~V} \AA^{-1}$ region and saturation behavior in $0.4-1.2 \mathrm{~V}^{-1}$. The dotted curve is drawn to guide the eye.

3.5 The plot of the I-V characteristics on Fowler-Nordheim scales. The fact that the graph is not a straight line, illustrated by the two fitted dotted lines (drawn to guide the eye) with different slopes, shows the non Fowler-Nordheim behavior. Section (A) is the high electric field regime and section $(\mathrm{B})$ is the low electric field regime. . . . . . . . . . . 46

3.6 Potential profile along the the center of the SWNT in the turn-on regime. Dotted line is the HOMO level of the structure at: (A) 0.2, (B) 0.25, (C) 0.3, (D) 0.35 , and (E) $0.4 V \AA^{-1} \ldots \ldots . . . . . . .446$

3.7 Potential profile along the the center of the SWNT in the saturation regime. Dotted line is the HOMO level of the structure at: (A) 0.6, (B) 0.75, (C) 0.9, (D) 1.05 and (E) $1.2 V \AA^{-1}$. . . . . . . . . . 47

3.8 Potential barrier width between the potential well and vacuum at the HOMO energy level, circular dots represents the width at the low electric fields (corresponding to Figure 3.6) and square dots represent the width at the high electric fields (corresponding to Figure 3.7). . .

A.1 CVD setup consisting of a tube furnace and a quartz tube. . . . . . . 57

A.2 The manifold setup where precise flows of the gases are mixed. . . . . 57

A.3 SEM images using a FE Hitachi S4700 SEM of: (A) CNTs grown from catalyst islands (B) Zoomed in image of the catalyst islands and CNTs (C) A long CNT grown from a catalyst island towards an electrode. . 58 
A.4 SEM images using a Philips 505M SEM of: (A) CNT forests grown from patterned electrode (field of view of $2 \mathrm{~mm}$ ) (B) Zoomed in image of CNT forests grown from patterned electrode (field of view of $1.5 \mathrm{~mm}$ ) (C) TEM image of the grown MWNT using an FEI Titan TEM. . . . 59

A.5 Reassembled and working Philips 505M SEM. . . . . . . . . . . . . 60

A.6 SEM images using a Philips 505M SEM of: (A) Carbon fibers (B) A canadian penny $(\mathrm{C})$ a pealed electrode. . . . . . . . . . . 60

A.7 Assembled and working UHV chamber. . . . . . . . . . . . . . 61 


\section{Dedication}

I want to dedicate this work to my parents. 


\section{Statement of Co-Authorship}

Chapters 1, 4, and Appendix A were written by the author. Parts of chapter 1 are from the introduction of a published review paper co-authored with my supervisor, Dr. Alireza Nojeh. The rest of this review paper is presented in chapter 2. The structure of this paper was set by Dr. Nojeh but the literature search and summary compilation of many different papers were performed equally by Dr. Nojeh and I. Chapter 3 is a paper submitted for publication, which is co-authored by myself, Dr. Konrad Walus, and Dr. Alireza Nojeh. I performed the derivation, simulation, and analysis; I generated and prepared the figures; I wrote the first draft of the paper, and participated in the collaborative effort that led to the final version of the paper. 


\section{Chapter 1}

\section{Introduction}

\subsection{Motivation}

Electron beams play a very important role in many areas of science and technology, ranging from applications in everyday life such as cathode ray tubes in traditional television sets, to usage in well-established research instruments such as electron microscopes, to cuttingedge research in sophisticated scientific experiments in synchrotrons. Since the associated wavelength of an electron is very small (even sub angstrom) for electrons with kinetic energies of several kilo electron volts $(\mathrm{keV})$ that are easily achievable in laboratory settings, they can be much more easily focused compared to light beams for applications where a high spatial resolution is needed, such as micro and nano probing and pattern writing. Much progress has been made over the last several decades in electron-beam research, both in terms of electron emission from source, as well as beam optics. Current state-of-the-art electron microscopes can easily achieve an imaging resolution of a few nanometers and are routinely used in research (Figure 1.1). However, much work still remains to be done in this area. In particular, in many current electron-beam applications, the performance bottleneck is the electron source, i.e. the tip that emits the electrons into vacuum, where they subsequently undergo deflection, focusing, etc. based on the application. For the source, some of the major considerations include being able to generate a high enough current from a very small spot with little noise and fluctuation over extended periods of time. Also, it is important that the generated electrons be very similar (in terms of their energy), in order for the electron-optical system to direct all of them in a similar fashion. 


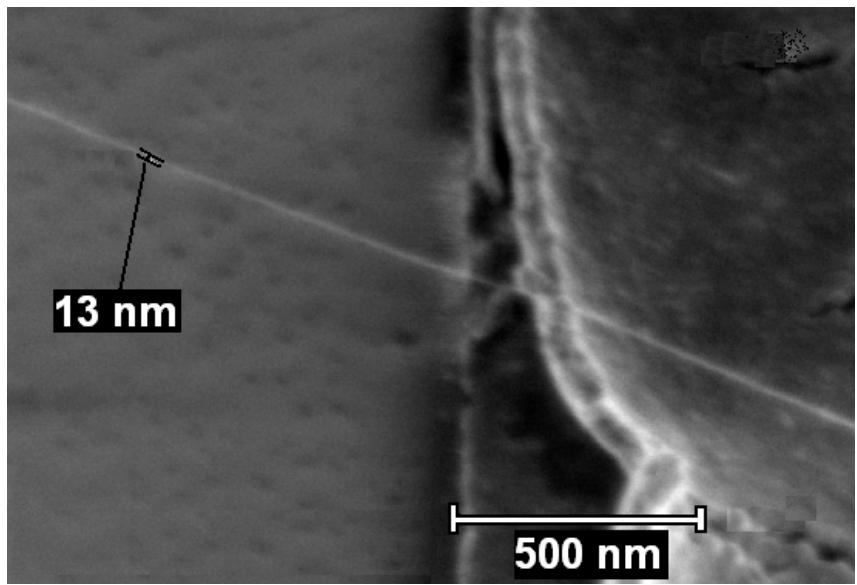

Figure 1.1: A high-resolution image of a carbon nanotube suspended over the edge of a peeled metal electrode, obtained in an FEI Sirion scanning electron microscope.

Thus, the field of electron emission is a very active area of research and many scientists and engineers around the world are on the quest for new, improved electron sources (the terms source and emitter are used interchangeably in this thesis). Due to their outstanding electronic and mechanical properties, carbon nanotubes, allotropes of carbon with nanoscale diameters, have attracted much attention during the last decade and a half. One of the main applications they are being researched for is electron emission. Carbon nanotubes are found to be an ideal candidate for electron emission due to their physical properties (further discussed in chapter 2). However, the exact nature of their electron emission behavior, which deviates from the traditional Fowler-Nordheim model, is a much debated topic in the literature (also discussed in chapter 2). The third chapter of this thesis will address this problem using a fully first-principles approach. Also in the course of this work, I have built several equipment and developed various recipes for growing carbon nanotubes, in order to set the stage for further experimental characterization of nanotube electron sources. These will be introduced briefly in Appendix A. 


\subsection{Thesis Overview}

This thesis comprises two journal papers. Chapter 2 is a published review paper in Modern Physics Letters B [1], which provides a brief review of electron emission and an introduction to carbon nanotubes. It also provides an overview of the subject of electron emission from carbon nanotubes with an emphasis on the discrepancy that exists in the literature in recognizing and reporting the current saturation behavior among carbon nanotube-based electron emitters. In this work, the most recent theoretical work on carbon nanotube emitters including the modeling challenges that exist with using first-principles are also presented.

The third chapter, which is a submitted paper, is a first-principles study of field electronemission. In this work using a fully first-principles approach, the quantum tunneling problem of a single-walled carbon nanotube is solved in three-dimensions using the nonequilibrium Green's function in a new way. This work confirms the saturation behavior observed in experiments and explains the reason behind this saturation mechanism. The presented method can also be generalized into a generic transport solver that can be used to solve any nano-scale transport problem using a first-principles approach. It has the flexibility to be tied into any first-principles software package (i.e. Gassuain, Siesta, etc.) and is also capable of including vacuum in the Hamiltonian, which the existing commercial transport solvers like TranSiesta or Qunatum Wise (previously known as Atomistix) do not model.

Appendix A of this thesis will discuss the experimental apparatuses built for characterizing electron emission from carbon nanotube electron sources. 


\section{Bibliography}

[1] P. Yaghoobi and A. Nojeh. Electron emission from carbon nanotubes. Modern Physics Letters B, 21(27):1807-1830, 2007. 


\section{Chapter 2}

\section{Electron Emission From Carbon Nanotubes}

\subsection{Electron Emission Mechanisms}

There are various well-established mechanisms through which electrons can leave a source and emit into vacuum. In thermionic emission, the source, typically a metal with a high melting point such as tungsten is heated to very high temperatures, usually around 3000 $\mathrm{K}$, and thus some of the electrons gain enough kinetic energy to simply overcome the workfunction barrier and leave the Fermi level for the vacuum level (Figure 2.1(a). A small externally applied electric field will then pull these electrons in the desired direction. In field-electron emission, or field-emission (FE) for short, a very high external field (on the order of a few volts per nanometer or higher) is applied to the source, such that the vacuum level is significantly lowered just outside the surface and only a thin energy barrier remains between the electrons and vacuum. Thus, through the process of quantum-mechanical tunneling, some of the electrons directly go to vacuum without having to acquire enough energy to overcome the barrier (Figure 2.1(b). This phenomenon was modeled by Fowler and Nordheim in 1928 [1] and their model is still in widespread use in studying field-electron emitters. A combination of relatively strong external fields (less than those required for field-emission) and high temperatures (less than those required for thermionic emission) can also lead to the so-called Schottky emission (Figure 2.1(c)). Another emission mechanism, which is widely studied, is photo-electron emission, or photo-emission for short, where

A version of this chapter has been published. Yaghoobi, P. and Nojeh, A. (2007) Electron emission from carbon nanotubes. Modern Physics Letters B. 21:1807-1830. 
photons with energies higher than the workfunction of the electron source illuminate it and transfer enough energy to the electrons to overcome the emission barrier, similar to thermionic emission (Figure 2.1(d)).
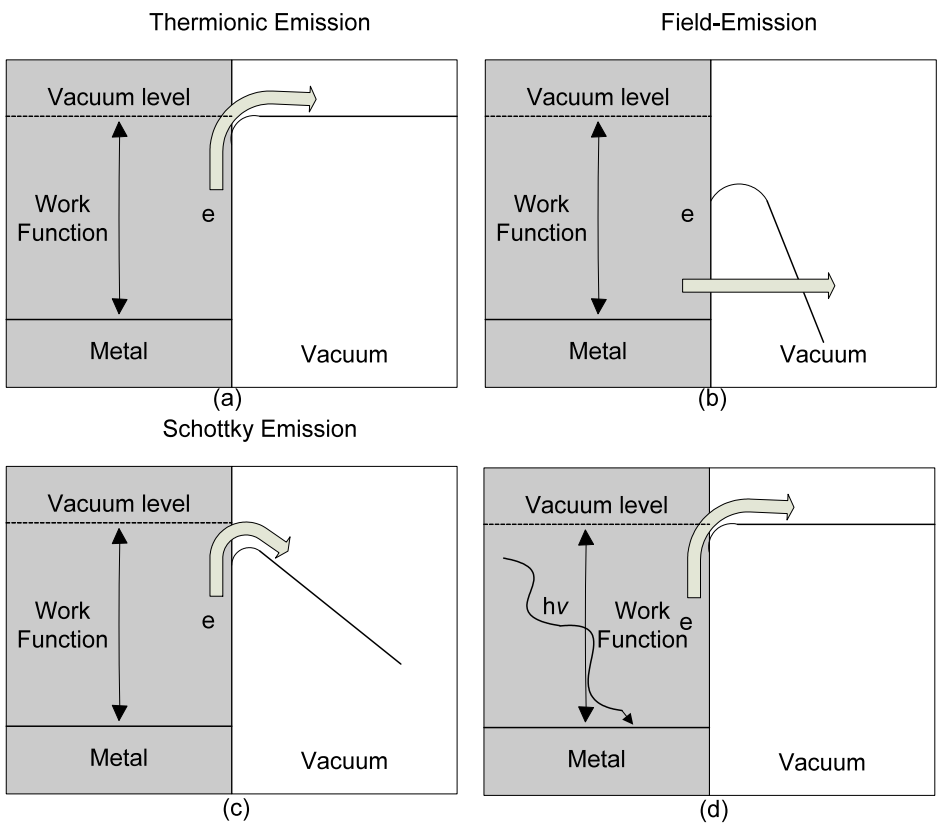

Figure 2.1: Various electron emission mechanisms: a) thermionic emission, b) fieldemission, c) Schottky emission and d) photo-emission.

Several parameters determine how an electron emitter will perform in an electron-optical system. Of particular importance are the brightness, energy spread, noise, stability and lifetime. Brightness is the amount of current density that the source can emit into the unit spatial solid angle, and directly affects how "sharp" a beam will be formed. This imposes a limit on resolution in lithography and microscopy by affecting not only the signal-tonoise ratio in detection, but also the beam spot at the focal plane due to geometrical aberrations. The energy spread of the emitted electrons affects the beam spot at the focal plane, and therefore the resolution, through chromatic aberrations: the higher the variety of the electron energies in the emitted beam, the harder for the electron-optical system to focus all the electrons to the same spot. Noise, such as shot noise due to the random emission of electrons in time, can have a significant impact on not only the signal-to-noise 
ratio in applications such as microscopy, but also the accuracy of the delivered dose in lithography. Finally, the stability of the emitted current over time and the lifetime of the electron source are also important considerations.

\subsection{Carbon Nanotubes}

Carbon nanotubes (CNTs) consist of one or more rolled layers of graphene, which is a sheet of carbon atoms with a thickness of one atom arranged in a hexagonal lattice like a chicken wire. The direction in which the graphene sheet must be wrapped to create a given nanotube is represented by a vector $(n, m)$ called the chiral vector. The integers $n$ and $\mathrm{m}$ denote the number of unit vectors along two directions in the honeycomb crystal lattice of graphene. If $\mathrm{m}=0$, the nanotube is called "zigzag". If $\mathrm{n}=\mathrm{m}$, the nanotube is called "armchair". Otherwise, it is called "chiral" (Figure 2.2).

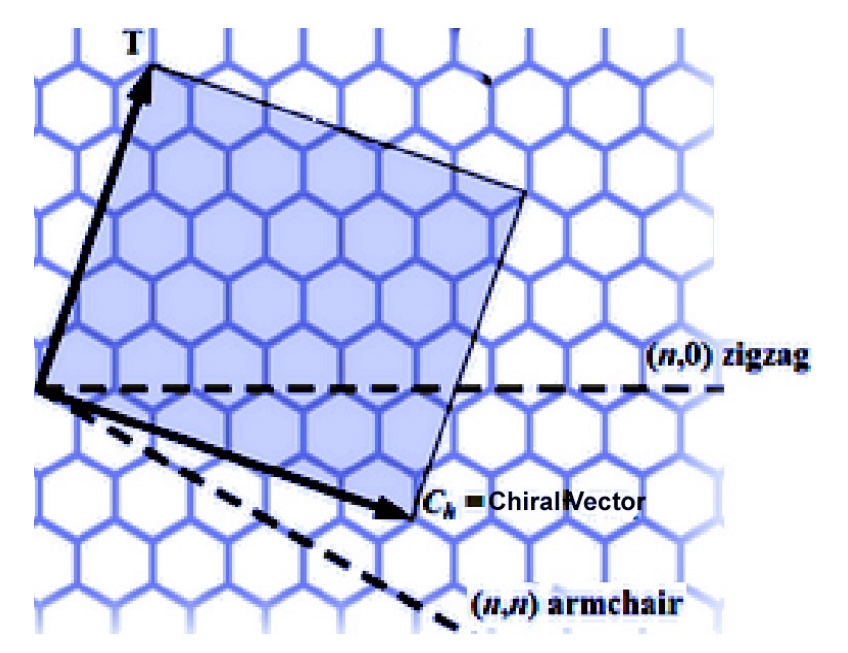

Figure 2.2: The hexagonal lattice of graphene and the unit vectors - rolling the graphene up along different directions will lead to nanotubes with various chiralities.

The chemical bonding between the neighboring atoms in CNTs is of $s p^{2}$ hybrid type, similar to that of graphite. This bonding structure, which is even stronger than the $s p^{3}$ bonds found in diamond, provides the molecules with their unique strength. Thus, CNTs 
are among the strongest and stiffest materials known, with an elastic modulus in the TPa range. Combined with their low density of $1.3-1.4 \mathrm{gcm}^{-3}$, this makes them suitable for very high strength to weight ratio applications. Based on its geometry, namely its chirality and diameter, a CNT can be metallic or semiconducting. With lengths of up to millimeters demonstrated experimentally, CNTs have very high aspect ratios, virtually making them one-dimensional, leading to high transmission and even ballistic transport for electrons. Together with their ability to carry current densities of up to $10^{9} \mathrm{Acm}^{-2}$ (orders of magnitude higher than copper and silver), this makes them very attractive for electronic applications.

CNTs can be fabricated with various methods, most notably arc discharge, laser ablation and chemical vapor deposition. In arc discharge, a high discharge current blows carbon atoms out of a graphitic target (that could also contain catalytic metal) in a chamber. The carbon atoms then deposit in the form of nanotubes on a substrate. CNTs were first discovered by this method [2]. In laser ablation, a pulsed laser vaporizes the graphitic target in a high-temperature reactor in the presence of an inert gas. As the laser ablates the target, nanotubes are formed and carried by gas flow to a cooler surface in the reactor. This method has a yield of about 70\% [3]. Chemical vapor deposition (CVD) consists of decomposing hydrocarbons using high temperature and in the presence of catalyst material, and having the extracted carbon form nanotubes emanating from the catalyst island. It is a very common method for device fabrication since it allows one to directly grow CNTs in desired locations (pre-patterned catalyst islands) as opposed to other methods, which require the collection of CNTs after their growth and placing them in desired spots on a substrate. The gases commonly used for CNT growth are: a carrier gas such as hydrogen or argon and carbon containing gases such as ethylene or methane. The reaction temperature is usually in the $700-900{ }^{\circ} \mathrm{C}$ range. The catalyst material primarily consists of transition metal nanoparticles.

Due to their sharp geometry (high aspect ratio and small tip radius), which leads to significant enhancement of an externally applied electric field, ability to carry extremely high current densities, mechanical strength and chemical stability, CNTs seem like ideal candidates for electron emission applications. Moreover, their one-dimensionality and the strong presence of quantum effects in them create interesting opportunities for unusual emission characteristics that could be exploited for many novel applications. Good review 
articles on carbon nanotubes have been published in the past also. A good example is one by de Jonge and Bonard [4].

\subsection{Field-electron emission from individual nanotubes}

In 1928, R. Fowler and L. Nordheim proposed a model for electron emission from metals in an intense electric field. The model assumes that the source has a metallic density of states, the material is at ordinary temperature, and electrons face a one dimensional potential barrier. This model leads to a current density equation of the form:

$$
J=a E^{2} \exp \left[-\frac{b}{a}\right]
$$

where $\mathrm{a}$ and $\mathrm{b}$ are constants. Generally for emission from a metallic emitter, equation 2.1 is graphically expressed on $\ln \left[I E^{-2}\right]$ versus $E^{-1}$ scales, which would lead to a straight line.

Currently, carbon nanotubes are intensively studied for field-emission. It has widely been assumed that because of their metallic or semi-metallic properties their field-emission behavior can be explained by the Fowler-Nordheim (FN) model. In this section and section 5, some of the research on field-emission from individual and collections of CNTs is discussed, with a strong emphasis on to what degree the FN model could be applicable. Although current saturation at high fields in CNTs is a generally accepted behavior, both linear and non linear FN curves are presented in the literature.

\subsubsection{Single-Walled Carbon Nanotubes}

SWNTs have been investigated by many researchers for their field-emission (FE) properties. Due to the irreproducibility of their growth and thus performance, their practical applications have been minimal at best; however, their excellent properties have enticed researchers to investigate their emission properties in detail. 


\subsection{Field-electron emission from individual nanotubes}

One of the most noted properties of FE from CNTs is its current saturation behavior at high fields [4-9]. Dean et al. demonstrated this by setting up a SWNT in a point-to-plane electrode geometry and performing FE experiments in a field-emission microscope (FEM). Figure 2.3 illustrates their results and shows a reversible current saturation behavior. This behavior would lead to a non-linear FN plot, and was explained by the authors to be caused by contact resistance, vacuum space charge, and most importantly, adsorbates $\left(\mathrm{H}_{2} \mathrm{O}\right.$ in their experiments). As a result of field and current-induced decrease in the tunneling enhancement of the adsorbate states, adsorbates saturate the current.

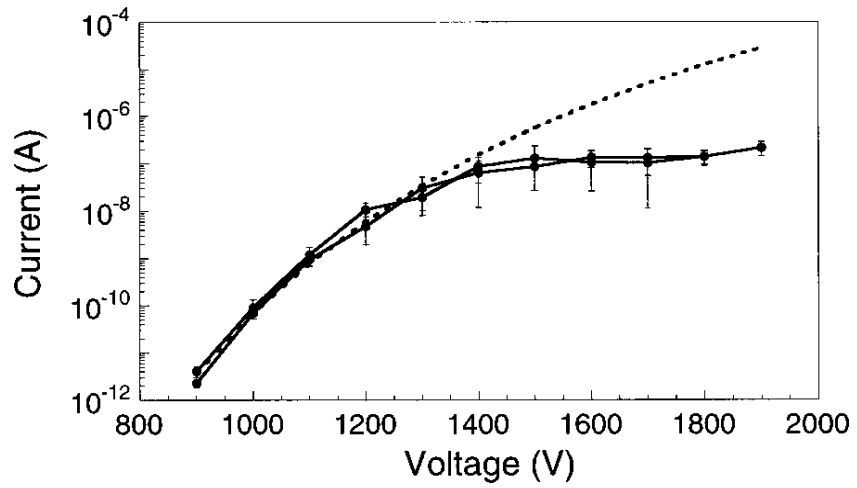

Figure 2.3: The current saturation behavior of an individual SWNT - the dashed line is a FowlerNordheim equation fit to the low voltage data [10].

In our own experiments on FE properties of SWNTs lying on a dielectric (Figure 2.4 (a)), a non-linear FN curve was observed (Figure 2.4 (b)) [11]. We believe this non-linearity to be partially due to the fact that the FN formulation is for a flat metal surface and cannot be directly applied to a complex 3-dimensional structure like a CNT on a surface. Also, the density of states at the tip of the CNT is not necessarily metallic and, as shown by Zheng et al., there could exist a nanoscale potential well at the tip that significantly affects the electronic structure [12]. 


\subsection{Field-electron emission from individual nanotubes}

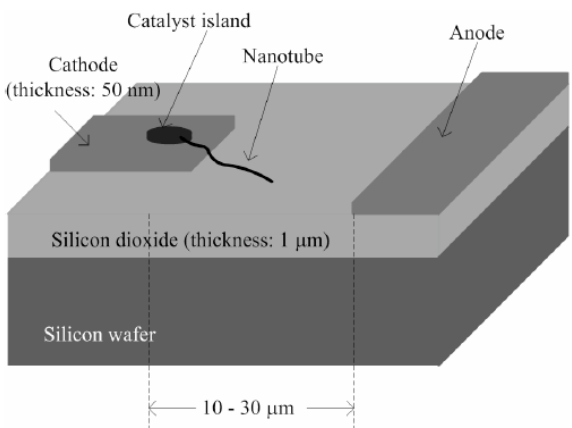

(a)

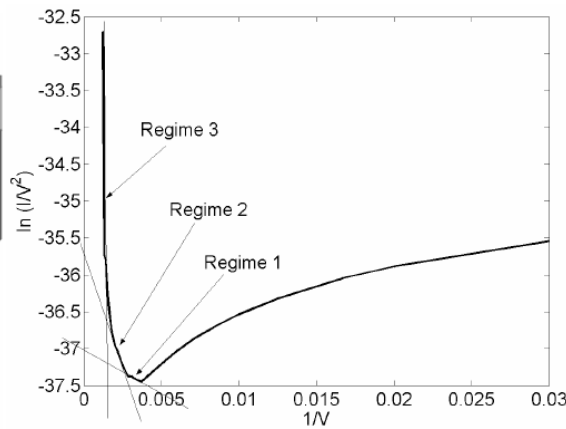

(b)

Figure 2.4: (a) Schematic of a surface nanotube electron emitter. (b) Emission current vs. applied voltage potted on an FN scale [11].

\subsubsection{Multi-Walled Carbon Nanotubes}

Bonard et al. investigated the emission from individual MWNTs by isolating them from a MWNT film using a sharp anode (Figure 2.5 (a)) [13]. Similar to SWNTs, MWNTs show a current saturation behavior (Figure 2.5 (b)).

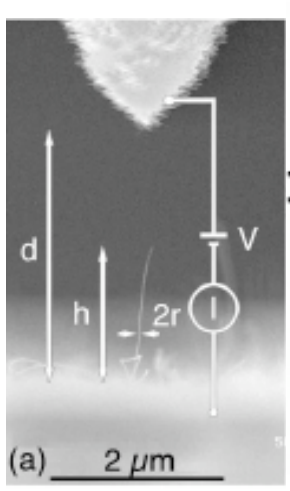

(a)

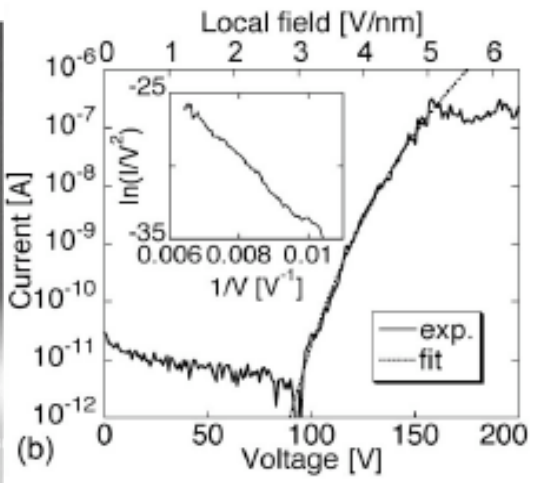

(b)

Figure 2.5: (a) Scanning electron micrograph of a nanotube of length $\mathrm{h}=1.4 \mathrm{um}$ and radius $\mathrm{r}=7.5 \mathrm{~nm}$ with a sharp anode positioned at a distance $\mathrm{d}=2.65 \mathrm{um}$. (b) The corresponding I-V curve with a fit to the FN law in the dotted line. The FN plot is given in the inset [13]. 


\subsection{Field-electron emission from individual nanotubes}

Tanaka et al. investigated FE from a stand-alone MWNT [14]. They deposited amorphous carbon on its tip to create an insulating layer. They performed electron emission experiments and then they removed the insulating layer by using heat treatment. When the insulating layer was removed, the turn on voltage decreased by $200 \mathrm{~V}$, and the increase rate of current with voltage became higher. The I-V characteristics of these MWNTs are shown in Figure 2.6 (a) for before and after heat treatment. A current saturation behavior is apparent, and the corresponding FN plot deviates from a single straight line as shown in Figure 2.6 (b).

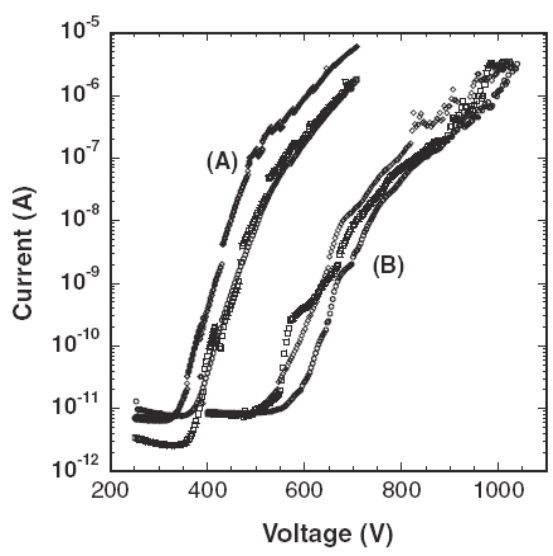

(a)

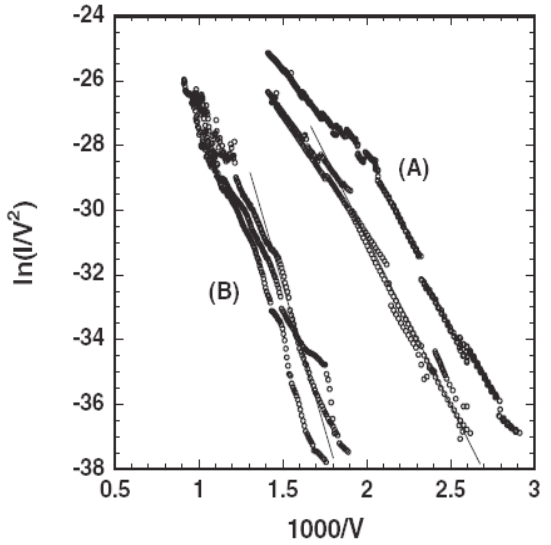

(b)

Figure 2.6: (a) IV characteristics of a MWNT with amorphous carbon on tip before (Group B) and after (Group A) the heat treatment. For the heat treatment, the tungsten tip was heated to $700 \mathrm{~K}$ during the field emission at $800 \mathrm{~V}$. (b) FN plot of the data with straight line fits in lower voltage regions [14].

$\mathrm{Xu}$ et al. performed in-situ electron emission experiments from an individual MWNT in a transmission electron microscope (TEM). They investigated both the field enhancement factor [15] and the work function [16]. They varied the distance between the MWNT and the anode and measured the field enhancement factor. The measured I-V characteristics and FN plots are shown in Figure 2.7. Their results show a linear FN behavior and also a non-saturating I-V plot.

For calculating the nanotube work function, they resonated a MWNT to its mechanical resonance by applying an alternating electric field and then adding a DC voltage to the 


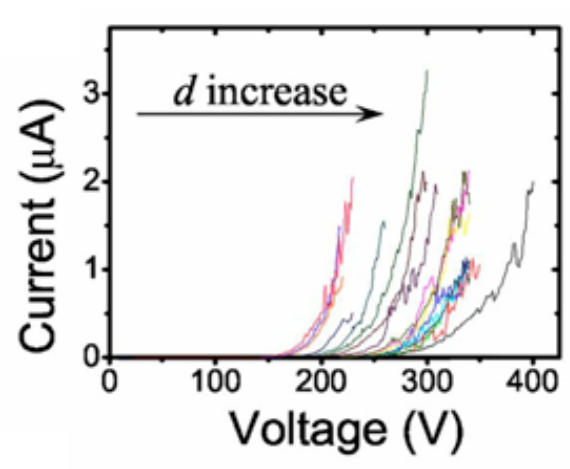

(a)

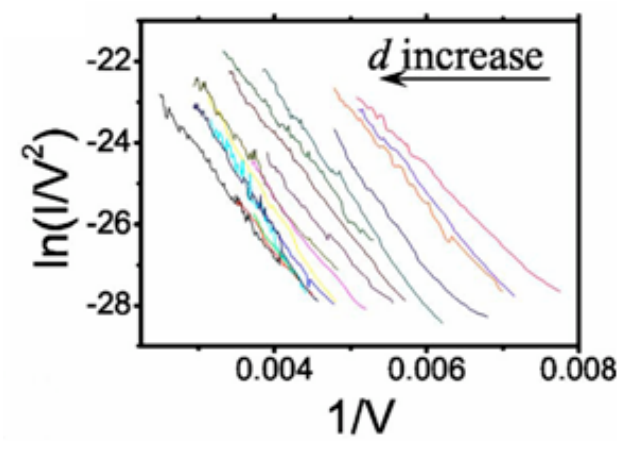

(b)

Figure 2.7: (a) I-V curves of the field emission for different nanotube-anode distances d. (b) The corresponding F-N plots [15].

alternating voltage. According to principle of contact potential difference, the DC voltage at which the MWNT stops resonating is the work function of the nanotube tip. By being able to calculate an accurate work function, they were able to fit a more accurate FN relationship to their experimental results (shown in Figure 9). The FN plot is again linear and no current saturation behavior is observed.

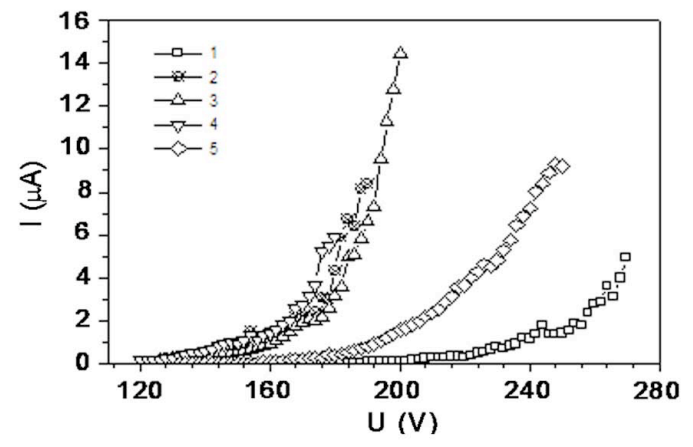

(a)

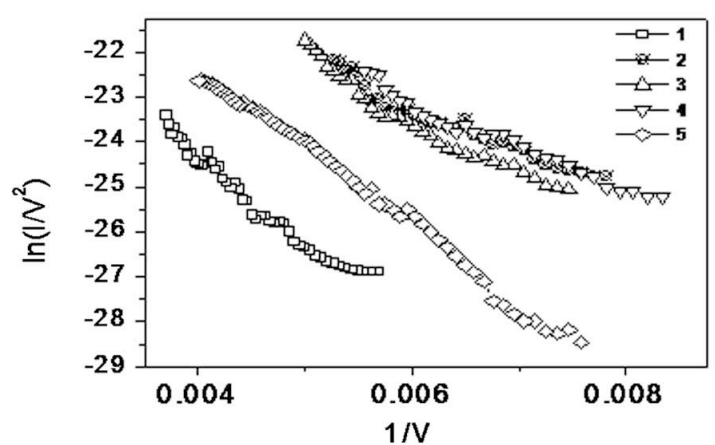

(b)

Figure 2.8: (a) I-V curves of 5 different nanotubes. (b) The corresponding F-N plots $[16]$.

One of the important performance factors of an electron source is its brightness. de Jonge et al. tested this property by using a MWNT as an electron source in a point projection 


\subsection{Field-electron emission from individual nanotubes}

microscope [17]. This setup allowed them to estimate the radius of the source by counting the number of visible fringes made by a sharp edge touching the beam from the side. With a known radius of source they calculated the reduced brightness of the MWNT to be an order of magnitude larger than the current state-of-the-art electron sources. They also measured the brightness vs. energy spread for the nanotube source (Figure 10) [18].

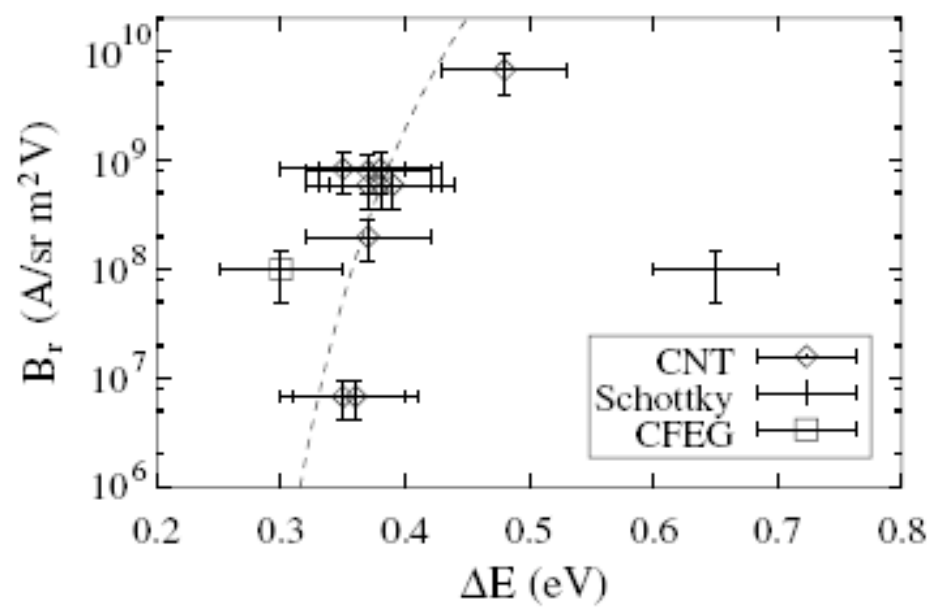

Figure 2.9: Brightness $(\mathrm{Br})$ as a function of energy distribution $(\Delta E)$ measured for several CNTs at currents of $10500 \mathrm{nA}$ and temperatures of $600700 \mathrm{~K}$. Calculated curve (dashed line). The data of the Schottky emitter and the tungsten cold field emission gun are also included [18].

In an atypical experiment, Chai and Chow investigated electron emission from the sidewall of a looped MWNT (Figure 2.10) [19]. Their result did follow the FN model. Interestingly, the FN data gave them a field-enhancement factor of about 400,000, which is much higher than is usually obtained for emission from a nanotube tip. As they point out, this result is completely counter-intuitive. Another way of fitting the FN data was the possible reduction in the nanotube work function due to the induced curvature. Their device fabrication process involved bombardment of the nanotubes by Ga ions to create the loop. They also speculate that the defects created by the ions on the sidewall might be at the root of the improved emission properties. Nonetheless, the emission characteristics were stable and reproducible. 

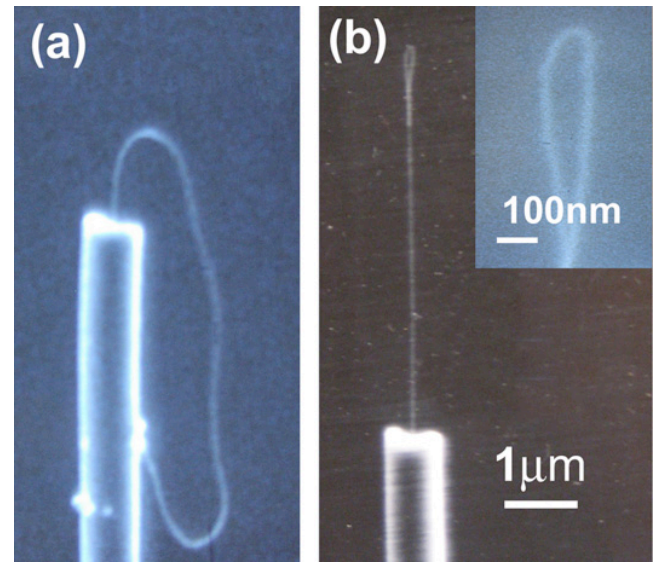

Figure 2.10: Nanotube sidewall emitter [19].

\subsection{Field-Electron Emission from Collections of Nanotubes}

\subsubsection{Single-Walled Carbon Nanotubes}

Collections of CNTs have more reproducible electron emission characteristics than individual CNTs since recreating exactly the same individual nanotube is difficult, whereas the average behavior of a large ensemble of nanotubes is reproducible. One of the major applications of collections of CNTs is in field-emission displays (FEDs). Various fabrication methods have been exploited in order to obtain the simplest and most reliable FEDs. Choi et al. demonstrated a triode type FED using SWNTs (Figure 2.11 (a)) [20]. The $\mathrm{I}-\mathrm{V}$ characteristics of this triode-type FED are shown in Figure 2.11 (b) and the current saturation behavior is apparent. Such behavior, as noted before, will lead to a non-linear FN curve. 


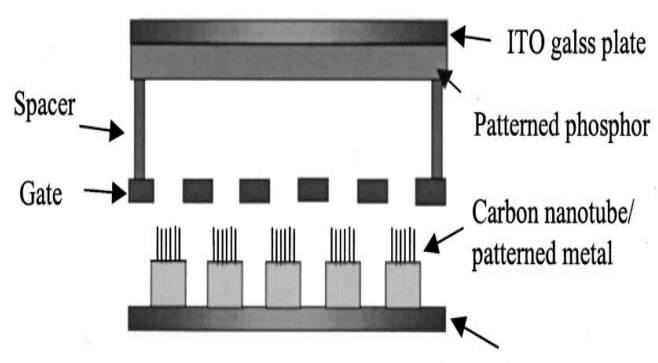

(a)

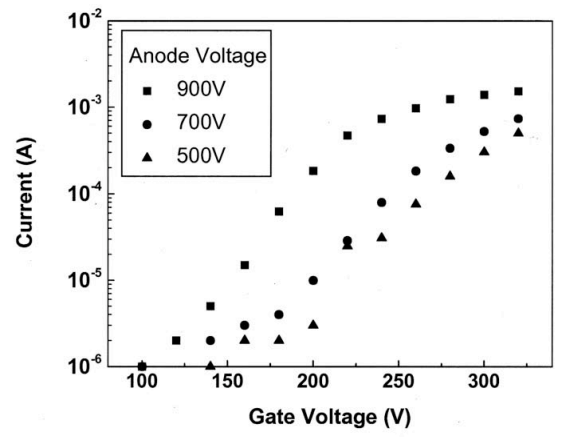

(b)

Figure 2.11: (a) Schematic geometry of a triode-type carbon nanotube-based field emission display. (b) Emission current of a triode type CNT-FED for various anode voltages. [20].

Lee et al. fabricated an organic flexible array of SWNTs by using self-assembled monolayers [21]. They did also observe current saturation behavior (Figure 2.12 (b)).

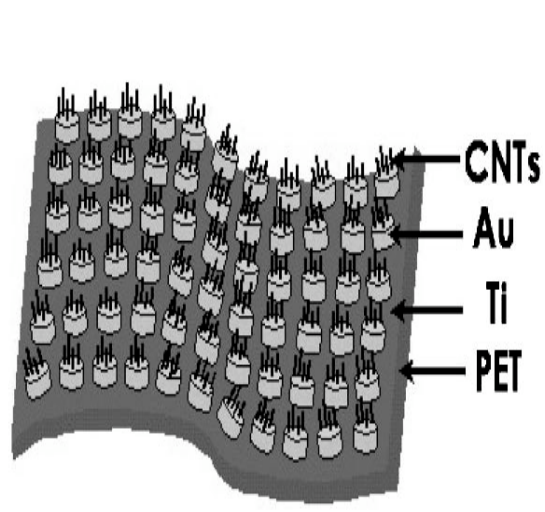

(a)

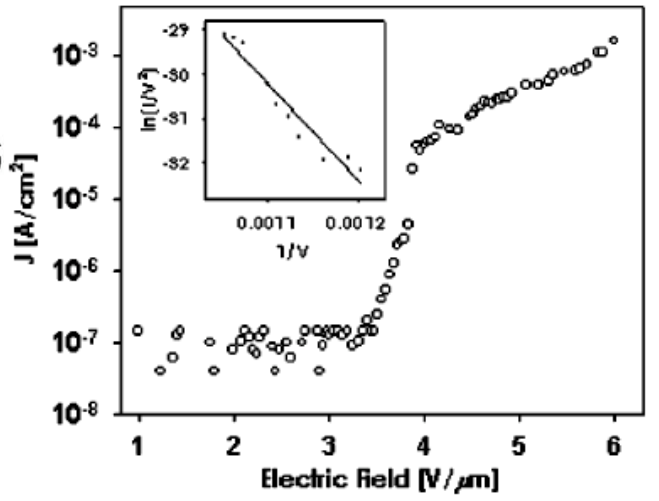

(b)

Figure 2.12: (a) schematic diagram of flexible substrate and aligned carbon nanotubes. (b) Emission current density vs. electric field for the emitter. Inset: FN plot $[21]$.

Bonard et al. also observed such current saturation behavior in their experiments on films of SWNTs (Figure 2.13) [6]. They attribute this to space charge effects, light emission 
coupled with field-emission, and difference in electron distribution between the tube and the tip. Zheng et al. have shown that at the tip where emission occurs, the local density of states presents sharp localized states that are close below the Fermi level and are dependant on the tip geometry [12]. The presence of these localized states could explain the current saturation behavior commonly noted in CNTs.

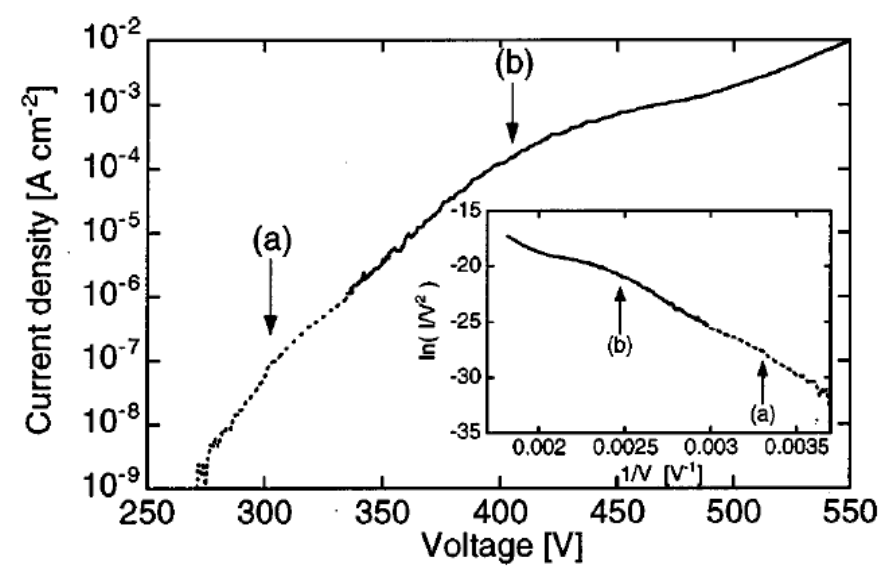

Figure 2.13: Single I V characteristics of a typical SWNT film. Inset: the corresponding FN plot. Arrows (a) and (b) indicate the end of the low current regime and the onset of the saturation, respectively [6].

\subsubsection{Multi-Walled Carbon Nanotubes}

Films of MWNTs produce the most reliable form of FE among the different configurations of CNTs. Collins et al. fabricated a film by using MWNTs made by arc discharge and mixing them with an epoxy [8]. This mixture was then dried by applying pressure. Their electron emission I-V curve is shown in Figure 15(a) for an onset voltage $\left(\mathrm{V}_{\text {ons }}\right)$ of $100 \mathrm{~V}$, where $\mathrm{V}_{\text {knee }}$ defines the point where the collected data deviates from low voltage behavior. The result was further analyzed by plotting the FN curve, where the non linear behavior is again noted (Figure $2.14(\mathrm{~b})$ ). 


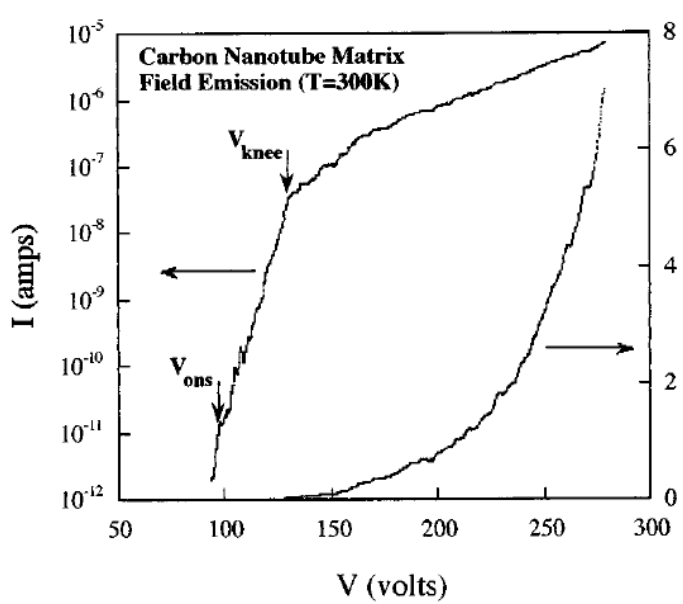

(a)

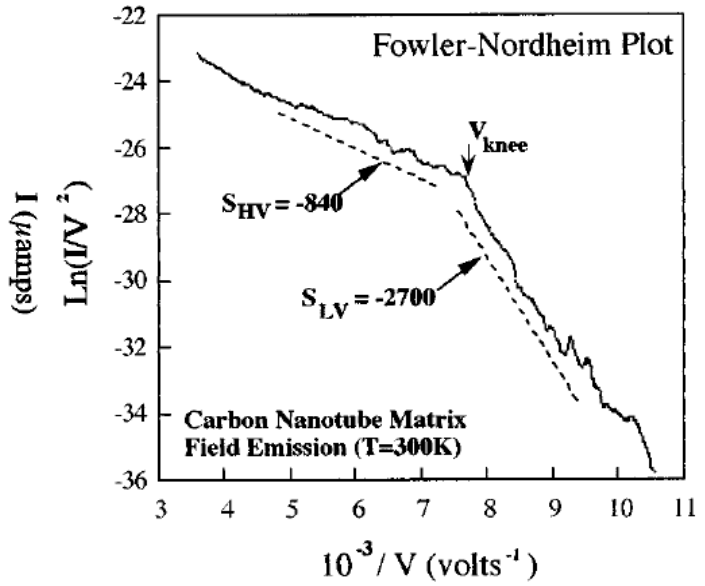

(b)

Figure 2.14: (a) I-V curve for field-emission from a MWNT matrix sample - it shows both logarithmic and linear current scales. (b) The corresponding FN plot [8].

This nonconformity to a single-straight-line FN behavior was said to be caused by the following reasons: 1) space charge surrounding the emission site would reduce the actual electric field at the tip at high current densities [22], 2) limited carrier concentration in a nonmetallic emitter could add to or exaggerate the space-charge effect, 3) nanotubes might have localized states at the tip that are weakly coupled to the bulk, 4) emission from multiple sources is not taken into consideration when using FN equations, 5) the non-emitting CNTs could create a large electric field that would create a complex field distribution configuration that is beyond the FN model, and 6) CNTs that are below the epoxy could cause dielectric breakdown at high electric fields, tunnel through, and emit electrons. This behavior was modeled by Latham and Wilson, who describe a situation where the electronic properties of the insulating layer dominate the I-V characteristics as opposed to the emitter itself [23].

Films of MWNT are also used for FEDs. Kwo et al. tested the characteristics of a FED by using such a film [24]. It was made by a printing process, where slurry of MWNTs was adhered to the electrode through heat treatment. The FE properties of the films were tested, and the emission I-V plot and the FN plot are shown in Figure 2.15. 


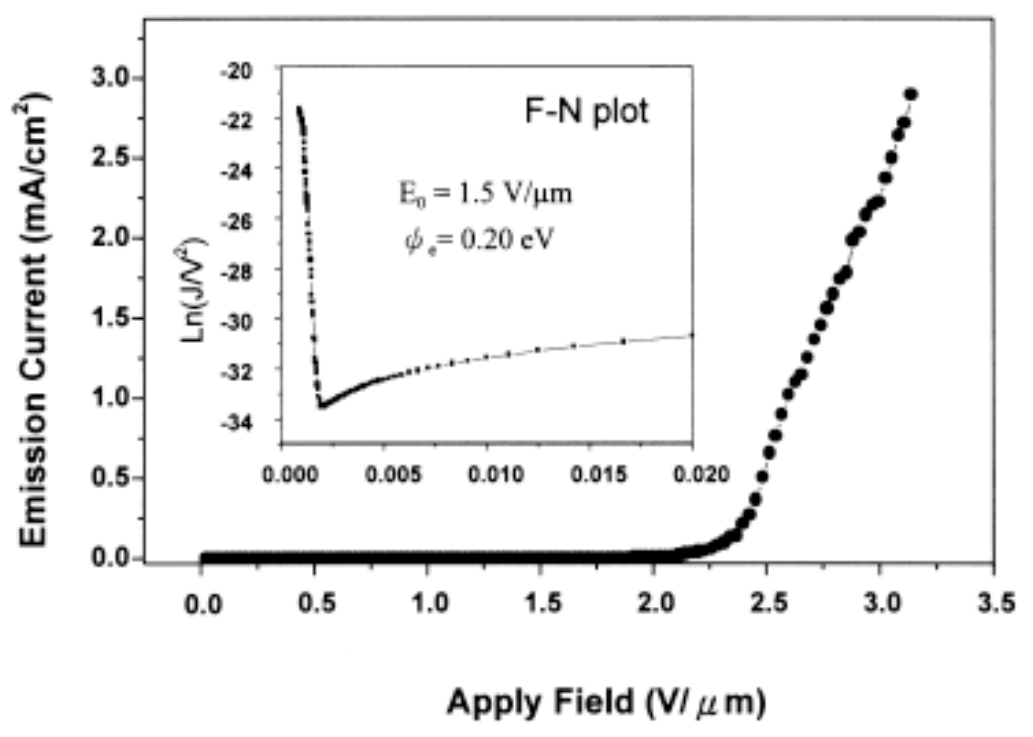

Figure 2.15: (a) IV characteristics of CNT clusters synthesized by arc discharge with $150 \mathrm{~A}$ arc current and $20 \mathrm{~V}$ arc voltage, under $500 \mathrm{mbar}(\mathrm{He})$; the inset shows the corresponding FN plot [24].

\subsection{Other Traditional Emission Mechanisms}

As discussed in section 2.1, field-emission is only one of several possible ways of extracting electrons from a source. Other mechanisms include thermionic emission, photo-emission, or a combination of any of those. Traditionally, field-emitters have provided higher-quality electron beams in terms of brightness and energy spread, and perhaps that is one of the reasons why there has been so much work on field-emission from nanotubes, and much less on other emission mechanisms. Nonetheless, we feel that the other mechanisms are also important and in this section shall briefly review some of the related work. In many cases, a combination of thermionic and field-emission was studied.

Chernozatonskii et al. investigated the effect of temperature on the FE of thin-film nanotube samples both single-walled and multi-walled. They observed that in the range of voltages before FE started, the emission behavior seems to follow the Richardson-Deshman- 
Schottky law for the temperature range under study (up to $745 \mathrm{~K}$ ) [25]. After FE started, they observed FN behavior adjusted for the effect of temperature (significantly increased).

The effect of direct heating and laser heating on FE of MWNTs was also investigated by Chen et al. [26]. They reported a factor of almost 50 times increase in field-emission current when the sample was heated from room temperature to $700 \mathrm{~K}$. They also noted a decrease in the turn-on field as a result of an increase in temperature. Koeck et al. also reported enhancement of about 30 times in the field-emission current of a CNT film with a temperature change from $380^{\circ} \mathrm{C}$ to $750{ }^{\circ} \mathrm{C}[27]$.

Cox et al. experimented with MWNTs grown by CVD at low temperature [28]. They believe that the low-temperature growth condition leads to defective nanotubes with poor thermal conductivity. When electric current was passed through these nanotubes, they observed electron emission from them when the amount of electric power dissipated in the nanotubes was in excess of $100 \mu \mathrm{W}$. Their estimate is that the peak thermionic emission happens at a nanotube temperature of around $2900 \mathrm{~K}$.

Another very interesting work on thermionic emission from nanotubes is that of Liu et al. [29]. They mounted a MWNT yarn in a hairpin configuration, much like a traditional tungsten thermionic emitter. They analyzed the luminescence spectra from the heated yarn to determine the temperature. At anode voltages below the onset of fieldemission, they attribute all the emission current to thermionic emission. Their emission curves have the same features as thermal FE from tungsten. By using Richardsons law, they determined the workfunctions of their samples to be in the 4.54-4.64 eV range. The full-width-at-half-maximum of the energy distribution of the emitted electrons was about $0.8 \mathrm{eV}$ at $2024 \mathrm{~K}$.

As discussed before, another mechanism to excite electrons out of a source is photoemission, where light particles transfer energy to electrons to overcome the workfunction barrier. The electron source in this case would be called a photocathode. Typically, since the workfunction of nanotubes is in the $4-5.5 \mathrm{eV}$ range, ultra-violet light is necessary to generate photo-electrons. One interesting experiment was done by Wong et al., where they studied photo-emission from a film of MWNTs [30]. They used wavelengths of $266 \mathrm{~nm}$, $355 \mathrm{~nm}$ and $532 \mathrm{~nm}$ to excite the emission. At low laser energy, with $266 \mathrm{~nm}$ (corresponding to a photon energy of $4.66 \mathrm{eV}$ ), they observed a one-photon assisted emission process. 
This was not the case with the other two wavelengths, which corresponded to photon energies significantly less than the nanotube workfunction. Even with $266 \mathrm{~nm}$, the quantum efficiency of the sample was small since, as explained by the authors, a large number of the nanotubes have workfunctions even higher than $4.66 \mathrm{eV}$. At high laser energies, they observed a notable rise in the emission signal as a function of laser energy for all three wavelengths, indicating a thermally-assisted field-emission process.

\subsection{Novel Electron Emission Mechanisms}

So far we have been focusing on more traditional methods of electron emission from nanotubes, namely field-electron emission, thermionic emission and photo-electron emission. In this section we will discuss an experiment where electron emission happens at least partially due to other mechanisms, which could possibly be quite particular to the nanotube structure.

A SWNT device was made where individual nanotubes were lying on a surface, connected to microfabricated electrodes to apply the field [31]. Electron emission experiments were performed inside an SEM. An external electric field, just below the threshold value necessary for field-emission, was applied to the device. Since the magnitude of the field was not high enough to drive electron emission, under these circumstances another agent was necessary to trigger the emission process. The primary beam of the SEM was used for this purpose. Thus, every time the primary beam scanned past the tip of the biased nanotubes, the tips emitted for a short period of time, leading to a momentary saturation of the secondary electron detector of the SEM and a bright spot at the nanotube tip on the image. Remarkably, although the nanotube tip is a very small area of a hollow structure and does not provide a bulk for interaction with the primary electrons, the electrons seem to interact very strongly with the tip, and the electron gain (from the primary to the emitted secondaries) was observed to be very high (estimated to be up to about 100). This beam-stimulated field-emission phenomenon was used to obtain a visual map of the emission hot-spots of a carbon nanotube emitter (Figure 2.16) to ensure that the nanotubes, and not other areas of the structure such as electrodes or catalyst material, are the active emitting parts of the device [32]. 

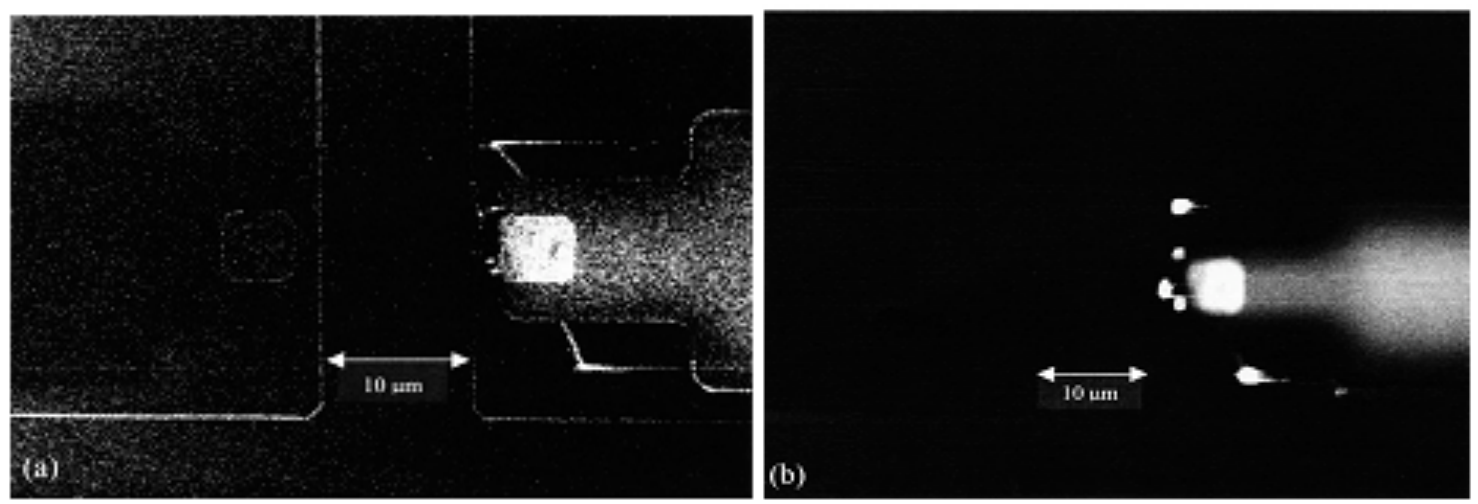

Figure 2.16: Bright spots at the nanotube tips as a result of beam-stimulated fieldemission. The wide semi-bright area on the right is the cathode electrode and the square is the nanotube growth catalyst island [32].

\subsection{Observation of Emission Sites}

One of the major difficulties in experiments and measurements on nanoscale devices arises from the fact that typically the main part of the device, such as a nanotube, is surrounded by larger, microscale, structures such as metal electrodes or catalyst islands that could severely affect the measurement results. In electron emission, this becomes particularly important since a sharp protrusion on the electrodes or catalyst can greatly contribute to the emission current, thus masking the real nanotube emission characteristics. It is thus extremely important to be able to visualize the emission hot-spots on a device.

Fujieda et al. performed electron emission from a MWNT inside a transmission electron microscope, where they where able to see a bright spot on the nanotube tip (Figure 2.17). Thus, they were also able to observe the correlation between structural damage at the emitting tip and large fluctuations happening at high emission currents [33]. They conjecture that the mechanism behind the formation of this bright spot is the interference between the primary electrons passing through the nanotube deflected due to field-emission and those passing outside of the nanotube unaffected by field-emission. 


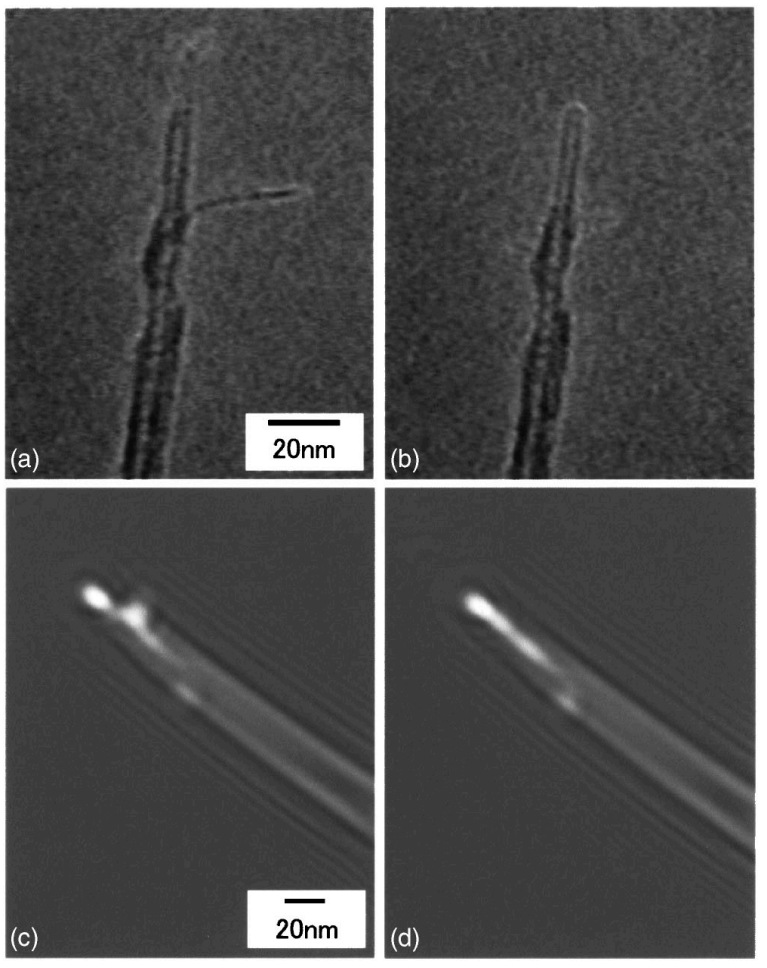

Figure 2.17: Bright emission spot at a nanotube tip emitting electrons, imaged in a transmission electron microscope, and structural damage to the nanotube tip [33].

Field-emission electron microscopy and photo-emission electron microscopy are well-established techniques to visualize electron emission from a surface. Gupta et al. used these techniques to look at the emission sites of both SWNT and MWNT matt samples [34]. By visualizing the emission hot-spots, they observed that although the surface contained more than $10^{8} \mathrm{~cm}^{-2}$ nanotubes, the emission site density was on the order of $10^{4} \mathrm{~cm}^{-2}$, making individual emission sites easily visible. By measuring the emission intensity from these sites, they studied the temporal stability and temperature dependence of emission, as well as the role of adsorbates in emission properties. By using a substrate heater, they studied the emission properties of the samples up to $1000{ }^{\circ} \mathrm{C}$. They obtained up to a 3 -fold increase in the integrated brightness by this temperature change, which could be related to both direct thermally-assisted emission, and the self-cleaning of the emission sites from chemisorbed molecules, induced by high temperature and field. 
Dean and Chalamala performed field-emission microscopy of samples containing SWNTs protruding from the surface for lengths of several microns [35]. They observed the emission patterns corresponding to individual nanotubes (Figure 19 (a)). The lobed patterns were essentially identical to those obtained as a result of adsorbate atoms and molecules on metallic field-emitters. By heating the sample to temperatures of up to 900K, adsorbates were removed and the lobed patterns disappeared, revealing the much dimmer emission patterns resulting from the nanotube itself (Figure 2.18 (b)). The emission current also had a significant drop. They speculate that the obtained images under these conditions do not correspond to individual atoms, but rather depict the surface electronic structure of the localized cap states of the nanotube.

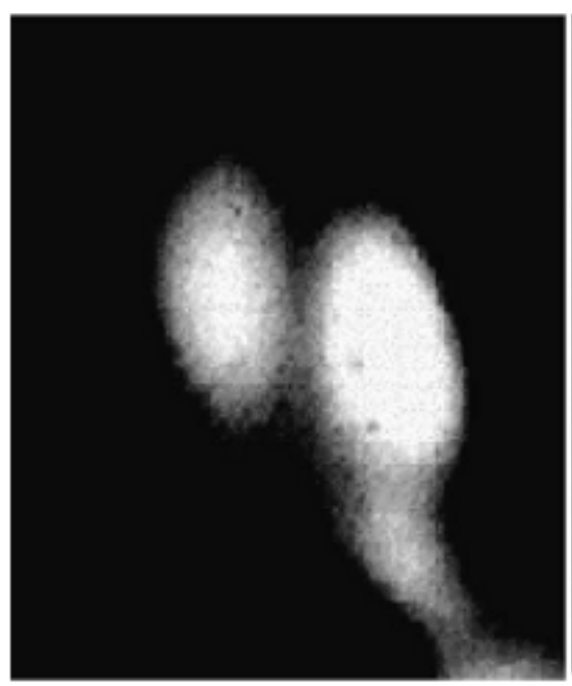

(a)

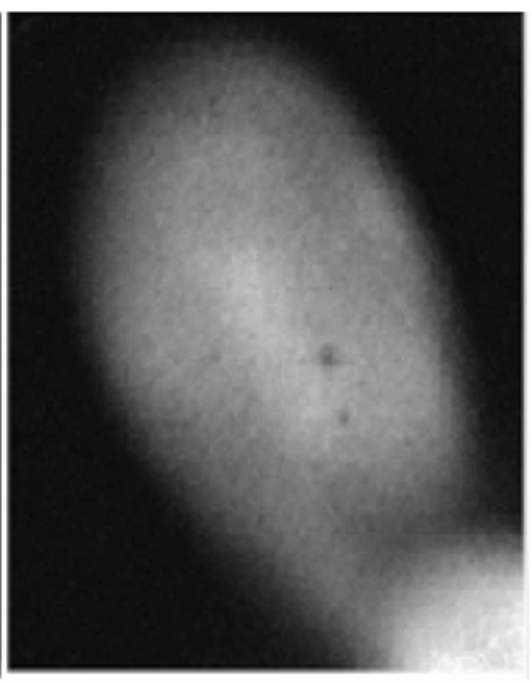

(b)

Figure 2.18: Electron emission patterns from a SWNT in a field-emission microscope [35].

Similar experiments were performed by Hata et al. on MWNTs [36]. Again, heat treatment was used to remove the adsorbates and reveal the emission patterns resulting from the nanotube tip. They observed streaks between the bright spots corresponding to adjacent emission sites on the nanotube tip, which they explained in terms of Youngs interference fringes. Similar results were obtained by Tanaka et al. [37], which they argue could be 
explained in terms of both interference and a standing wave along the circumference of the nanotube.

In addition to field-emission microscopy, Kuzumaki et al. also performed field ion microscopy of nanotube samples [38]. Thus, they were able to image both the electric field concentration sites and the surface work function. From their results it appears that the pentagonal carbon rings at the tip are not highly-emitting areas. Thus, they conjecture that the patterns observed in field-emission microscopy do not correspond directly to emission sites, but are rather the result of the interference of electrons being emitted from various sites as seen in field ion microscopy. They also performed emission experiments while looking at the nanotube tip inside a high-resolution transmission electron microscope, and observed that the nanotube tip becomes deformed and a protrusion is created, possibly by some of the atoms reconfiguring themselves from $s p^{2}$ to $s p^{3}$ bonding (Figure 20). Bonard and colleagues also reported field-emission microscopy images very similar to the ones mentioned above [39], and concluded that the electrons were emitted from localized states at the tip, and not de-localized electrons such as in metals.

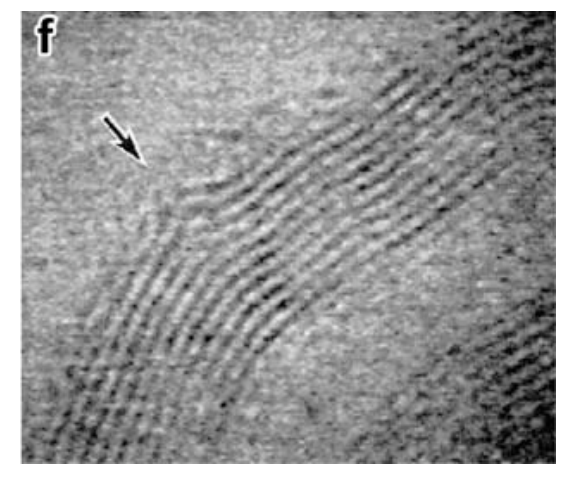

Figure 2.19: Deformed nanotube tip as a result of electron emission, seen in a transmission electron microscope [38]. 


\subsection{Modeling and Simulating Electron Emission From Carbon Nanotubes}

As for any nanoscale physical modeling and simulation problem, the theoretical study of electron emission from carbon nanotubes presents major difficulties. On one hand, for an accurate theoretical analysis, the system cannot be considered a continuous bulk and treated classically with continuum models and the exact atomistic nature of the structure must be taken into account. On the other hand, typically in a nanotube device there is a large enough number of atoms that solving the many-body quantum mechanical problem for the entire system is nearly impossible even with the most powerful computers of today. Thus, the art of modeling such systems necessitates being able to identify which aspects of a given problem need to be treated using atomistic simulations and what parts can be analyzed with more approximate, classical representations. Thus, usually a hybrid approach can be used to keep the problem manageable, while still capturing the essence of the nanoscale physics involved. One such hybrid method has been discussed by Han [40].

Currently, to the best of the authors' knowledge, a universal theory explaining all aspects of electron emission from carbon nanotubes does not exist. As seen in some of the previous sections, even in the experimental world there is still a debate in the research community on some of the fundamentals of this process, e.g., whether or not a nanotube emitter follows a traditional Fowler-Nordheim behavior. In this section we will review some of the theoretical works on electron emission from nanotubes to shed further light on the topic.

Zhou and Kawazoe performed first-principles calculations within the framework of the linear combination of atomic orbitals for molecular orbital (LCAO-MO) cluster model on a $(5,5)$ nanotube containing 18 unit cells (approximately $3.5 \mathrm{~nm}$ in length), capped at both ends [41]. Although this nanotube is theoretically metallic, their results showed that the electron emission from the SWNT is not a continuum as in metals, but is rather strongly dependent on the precise nature of the orbitals at the tip and the link between the tip and body. They also concluded that the emission characteristics could not be described by the FN model, and the experimental results showing such behavior are due to bundle effects, and not directly due to individual nanotubes. 
Kim et al. used the density functional theory to study capped $(5,5)$ and $(9,0)$ nanotubes under electric fields at the level required for field-emission [42]. For the (5,5) nanotube, although metallic in theory, they found that the effective workfunction decreased linearly with increasing the electric field, as opposed to the quadratic dependence typical in metals.

Zhou et al. studied field-emission from an open-ended $(6,6)$ nanotube, also metallic [43]. They used first-principles calculations with LCAO-MO. They found that besides the first row of carbon atoms directly in touch with vacuum, their first neighbors also contributed to the number of emitted electrons significantly.

Typically when performing first-principles calculations on nanotubes, due to the computationally expensive nature of the work, a short section of a nanotube is used, which cannot reproduce a realistic level of external field enhancement. Thus, it is customary to use a value for the applied field that already includes the field enhancement effect. A different approach was adopted by Luo et al., where they added extra negative charge to the short nanotube to generate the strong electric field [44]. They used the density functional theory to study the potential barrier near the tip. They observed higher transmission and better emission properties for capped nanotubes than open-ended ones (contrary to the observation made by Zhou and colleagues [43]).

Models that take the atomic nature of the nanotube into consideration and use firstprinciples type of calculations to study the electronic structure are quite limited in the size of the nanotubes they can handle. As such, in order to study nanotube emitters in the context of real applications, typically one has to resort to semi-classical, continuum models. An example of such a model is the work by von Allmen et al., where they approximated the nanotube with a conductive cylinder to study the field enhancement and emission properties of arrays of nanotubes for application in field-emission displays [45]. The transmission coefficient, however, was found by solving the one-dimensional Schrödinger equation. They found that there was an optimal nanotube separation/height ratio to achieve maximum current density from the array.

Shimoyama et al. also studied arrays of nanotubes by assuming that the nanotubes were continuous, conductive materials with hemispherical caps [46]. The dimensions of the nanotubes they studied corresponds to MWNTs. They found that the field strength at the 
nanotube tip decreased with increasing the nanotube diameter and tip radius of curvature. They also observed that the field strength was significantly reduced when the nanotube density in the array was more than $10^{9} \mathrm{~cm}^{-2}$ (corresponding to inter-nanotube distances of less than about $300 \mathrm{~nm}$ ). For nanotube densities less than $10^{8} \mathrm{~cm}^{-2}$ (inter-nanotube distance of more than 1 micrometer) they found that the field strength was independent of density.

Another very interesting work was done by Zheng et al. who studied FE from a micrometerlong $(5,5)$ SWNT [12]. In order to overcome the challenge of dealing with large numbers of atoms while performing quantum-mechanical calculations, they adopted a hybrid method where a section of the nanotube away from the tip was treated using molecular mechanics. In this way they were able to simulate a realistic experimental situation and field-enhancement was a natural outcome of the simulation (rather than incorporated manually). They observed that most of the field dropped over a region within about $5 \mathrm{~nm}$ of the tip, creating a steep potential well in that region. They obtained field-enhancement factors in the 300-1200 range, confirming previous belief. In contrast to the results of Kim et al. [42], they found that the emission barrier height was a non-linear function of the applied field.

Lan et al. took the study of nanotube emitters one step further by calculating electron trajectories from nanotube arrays in a display device and investigating the effect of the emitter bias conditions on the display resolution [47].

As discussed in section 7 in one experiment the nanotube was biased just below the fieldemission threshold and emission was triggered by shining another electron beam on the tip. Typically when an electron beam hits a bulk material it undergoes multiple scatterings and generates secondary electrons in the process. This can be modeled using a Monte Carlo approach, for instance. However, due to the small interaction volume of the beam with the nanotube tip (on the order of a cubic nanometer of a hollow structure), such models cannot explain the large number of emitted electrons in that experiment. In Ref. [48] the authors created a model to explain that electron-stimulated field-emission effect, where we performed first-principles calculations to study the behavior of the electronic orbitals at the nanotube tip in the presence of an external electron (representing a primary electron from the beam). We observed that one extra electron could shift the nanotube energy levels 
upward by as much as about $1 \mathrm{eV}$, thus significantly increasing the tunneling probability and transmission coefficient, leading to high induced field-emission.

A model for calculating both field and thermionic emission currents taking into account the band structure of the nanotube and using a triangular-shaped potential barrier at the tip was presented by Tang et al. [49]. They investigated the competition between those two emission mechanisms and also studied the energy distribution of the emitted electrons.

\subsection{Final Thoughts}

In this article we reviewed some of the important aspects of electron emission from carbon nanotubes, from both experimental and theoretical points of view. Obviously it was impossible to cover all the interesting works that have been done in this area in this brief review. We saw that although many interesting and valuable attempts have been made in both directions, there are still disagreements between various experimental results on important issues such as whether or not field-emission from nanotubes follows the traditional Fowler Nordheim behavior. As well, we are far from having a universal theoretical model describing all the aspects of electron emission from nanotubes. Nonetheless, nanotubes are starting to find their way into real applications. Good examples include the work of Yabushita et al., where they report a compact field-emission SEM with a MWNT bundle cathode [50], and that of Getty et al. where they report a field-emission electron gun based on nanotube film for use in a miniaturized reflectron time-of-flight mass spectrometer [51].

Although the overwhelming majority of the works have focused on field-emission, more recently there has been an increased attention to thermionic emission as well. Also, other emission mechanisms such as electron-stimulated field-emission have been observed in nanotubes, seemingly due to their particular nanoscale structure. We believe that more peculiar and exotic emission phenomena, with strong signatures from the quantum mechanical nature of nanotubes, could be possible since nanotube structures are so drastically different from traditional bulk metal emitters that we have been used to for a long time. Thus, as the quest for better nanotube-based electron emitters and their transfer to the world of real applications continues, in addition to the systematic study of field- and thermionic 
emission, it seems to be important to look for such novel mechanisms and phenomena that might lead to improved characteristics in emitters, as well as open up possibilities for new applications. 


\section{Bibliography}

[1] R. H. Fowler; L. Nordheim. Electron emission in intense electric fields. Proceedings of the Royal Society of London. Series A, Containing Papers of a Mathematical and Physical Character, 119(781):173-181, 1928.

[2] Sumio Iijima. Helical microtubules of graphitic carbon. Nature, 354(6348):56-58, 1991. 10.1038/354056a0 10.1038/354056a0.

[3] Nasreen G. Chopra, Lorin X. Benedict, Vincent H. Crespi, Marvin L. Cohen, Steven G. Louie, and A. Zettl. Fully collapsed carbon nanotubes. Nature, 377(6545):135-138, 1995.

[4] Niels de Jonge and Jean-Marc Bonard. Carbon nanotube electron sources and applications. Philosophical Transactions of the Royal Society A: Mathematical, Physical and Engineering Sciences, 362(1823):2239-2266, 2004. 10.1098/rsta.2004.1438.

[5] Philip G. Collins and A. Zettl. A simple and robust electron beam source from carbon nanotubes. Applied Physics Letters, 69(13):1969, 1996.

[6] Jean-Marc Bonard, Jean-Paul Salvetat, Thomas Stöckli, Walt A. de Heer, László Forró, and André Châtelain. Field emission from single-wall carbon nanotube films. Applied Physics Letters, 73(7):918-920, 1998.

[7] Xueping $\mathrm{Xu}$ and G. R. Brandes. A method for fabricating large-area, patterned, carbon nanotube field emitters. Applied Physics Letters, 74(17):2549-2551, 1999.

[8] Philip G. Collins and A. Zettl. Unique characteristics of cold cathode carbonnanotube-matrix field emitters. Physical Review B, 55(15):9391, 1997.

[9] Jean-Marc Bonard, Thomas Stöckli, Frédéric Maier, Walt A. de Heer, André Châtelain, Jean-Paul Salvetat, and László Forró. Field-emission-induced luminescence from carbon nanotubes. Phys. Rev. Lett., 81(7):1441-1444, Aug 1998.

[10] Kenneth A. Dean and Babu R. Chalamala. Current saturation mechanisms in carbon nanotube field emitters. Applied Physics Letters, 76(3):375-377, 2000. 


\subsection{Bibliography}

[11] A. Nojeh and R. F. Pease. Field-electron emission from single-walled carbon nanotubes lying on a surface. In Canadian Conference on Electrical and Computer Engineering, Vancouver, British Columbia, Canada, 2007.

[12] Xiao Zheng, GuanHua Chen, Zhibing Li, Shaozhi Deng, and Ningsheng Xu. Quantummechanical investigation of field-emission mechanism of a micrometer-long singlewalled carbon nanotube. Physical Review Letters, 92(10):106803-4, 2004.

[13] Jean-Marc Bonard, Kenneth A. Dean, Bernard F. Coll, and Christian Klinke. Field emission of individual carbon nanotubes in the scanning electron microscope. Physical Review Letters, 89(19):197602, 2002. Copyright (C) 2007 The American Physical Society Please report any problems to prola@aps.org PRL.

[14] H. Tanaka, S. Akita, L. Pan, and Y. Nakayama. Barrier effect on field emission from stand-alone carbon nanotube. Japanese Journal of Applied Physics, 43(no. 2):864-867, 2004 .

[15] Zhi Xu, X. D. Bai, and E. G. Wang. Geometrical enhancement of field emission of individual nanotubes studied by in situ transmission electron microscopy. Applied Physics Letters, 88(13):133107-3, 2006.

[16] Zhi Xu, X. D. Bai, E. G. Wang, and Zhong L. Wang. Field emission of individual carbon nanotube with in situ tip image and real work function. Applied Physics Letters, 87(16):163106-3, 2005.

[17] Niels de Jonge, Yann Lamy, Koen Schoots, and Tjerk H. Oosterkamp. High brightness electron beam from a multi-walled carbon nanotube. Nature, 420(6914):393, 2002.

[18] Niels de Jonge, Myriam Allioux, Jim T. Oostveen, Kenneth B. K. Teo, and William I. Milne. Optical performance of carbon-nanotube electron sources. Physical Review Letters, 94(18):186807-4, 2005.

[19] Guangyu Chai and Lee Chow. Electron emission from the side wall of an individual multiwall carbon nanotube. Carbon, 45(2):281-284, 2007. 
[20] W. B. Choi, Y. W. Jin, H. Y. Kim, S. J. Lee, M. J. Yun, J. H. Kang, Y. S. Choi, N. S. Park, N. S. Lee, and J. M. Kim. Electrophoresis deposition of carbon nanotubes for triode-type field emission display. Applied Physics Letters, 78(11):1547, 2001.

[21] Ok-Joo Lee and Kun-Hong Lee. Fabrication of flexible field emitter arrays of carbon nanotubes using self-assembly monolayers. Applied Physics Letters, 82(21):3770-3772, 2003.

[22] J. P. Barbour, W. W. Dolan, J. K. Trolan, E. E. Martin, and W. P. Dyke. Space-charge effects in field emission. Phys. Rev., 92(1):45-51, 1953.

[23] R. V. Latham and D. A. Wilson. The energy spectrum of electrons field emitted carbon fibre micropoint cathodes. J. Phys. D, 16:455-463, 1983.

[24] J. L. Kwo, Meiso Yokoyama, W. C. Wang, F. Y. Chuang, and I. N. Lin. Characteristics of flat panel display using carbon nanotubes as electron emitters. Diamond and Related Materials, 9(3-6):1270-1274, 2000.

[25] L. A. Chernozatonskii, Z. Ya Kosakovskaya, Yu V. Gulyaev, N. I. Sinitsyn, G. V. Torgashov, and Yu F. Zakharchenko. Influence of external factors on electron field emission from thin-film nanofilament carbon structures. In The eighth international vacuum microelectronics conference, volume 14, pages 2080-2082, Portland, Oregon (USA), 1996. AVS.

[26] Yi-Chun Chen, Hsiu-Fung Cheng, Yun-Shuo Hsieh, and You-Ming Tsau. Electron field emission properties of carbon nanotubes during thermal heating and laser irradiation. Journal of Applied Physics, 94(12):7739-7742, 2003.

[27] Franz A. M. Koeck, Yunyu Wang, and Robert J. Nemanich. Thermionic converters based on nanostructured carbon materials. In 3rd Symp on New Frontiers and Future Concepts, volume 813, pages 607-613. AIP, 2006.

[28] D. C. Cox, R. D. Forrest, P. R. Smith, and S. R. P. Silva. Thermionic emission from defective carbon nanotubes. Applied Physics Letters, 85(11):2065-2067, 2004.

[29] Peng Liu, Yang Wei, Kaili Jiang, Qin Sun, Xiaobo Zhang, Shoushan Fan, Shufeng Zhang, Chuangang Ning, and Jingkang Deng. Thermionic emission and work function 
of multiwalled carbon nanotube yarns. Physical Review B (Condensed Matter and Materials Physics), 73(23):235412-5, 2006.

[30] Wong Teh-Hwa, M. C. Gupta, and C. Hernandez-Garcia. Nanosecond laser pulseinduced electron emission from multiwall carbon nanotube film. Nanotechnology, 18(13):4 pp., 2007.

[31] A. Nojeh, W. K. Wong, E. Yieh, R. F. Pease, and H. Dai. Electron beam stimulated field-emission from single-walled carbon nanotubes. Journal of vacuum science and technology. B. Microelectronics and nanometer structures. Processing, measurement and phenomena, 22(6):3124-3127, 2004.

[32] Alireza Nojeh, Wai-Kin Wong, Aaron W. Baum, R. Fabian Pease, and Hongjie Dai. Scanning electron microscopy of field-emitting individual single-walled carbon nanotubes. Applied Physics Letters, 85(1):112-114, 2004.

[33] T. Fujieda, K. Hidaka, M. Hayashibara, T. Kamino, H. Matsumoto, Y. Ose, H. Abe, T. Shimizu, and H. Tokumoto. In situ observation of field emissions from an individual carbon nanotube by lorenz microscopy. Applied Physics Letters, 85(23):5739-5741, 2004 .

[34] S. Gupta, Y. Y. Wang, J. M. Garguilo, and R. J. Nemanich. Imaging temperaturedependent field emission from carbon nanotube films: Single versus multiwalled. $A p$ plied Physics Letters, 86(6):063109-3, 2005.

[35] Kenneth A. Dean and Babu R. Chalamala. Field emission microscopy of carbon nanotube caps. Journal of Applied Physics, 85(7):3832-3836, 1999.

[36] Koichi Hata, Akihiro Takakura, Kenji Miura, Akinori Ohshita, and Yahachi Saito. Interference fringes observed in electron emission patterns of a multiwalled carbon nanotube. Journal of Vacuum Science and Technology B: Microelectronics and Nanometer Structures, 22(3):1312-1314, 2004.

[37] H. Tanaka, S. Akita, L. Pan, and Y. Nakayama. Barrier effect on field emission from stand-alone carbon nanotube. Japanese Journal of Applied Physics, 43(no. 2):864-867, 2004 . 


\subsection{Bibliography}

[38] Toru Kuzumaki, Yasuhiro Horiike, Tokushi Kizuka, Takayuki Kona, Chuhei Oshima, and Yoshitaka Mitsuda. The dynamic observation of the field emission site of electrons on a carbon nanotube tip. Diamond and Related Materials, 13(10):1907-1913, 2004.

[39] J. M. Bonard, J. P. Salvetat, T. Stöckli, Forró L., and A. Châtelain. Field emission from carbon nanotubes: perspectives for applications and clues to the emission mechanism. Applied Physics A: Materials Science and Processing, 69(3):245-254, 1999.

[40] S. Han. Theoretical study on the field emission of carbon nanotubes. PhD thesis, Seoul National University, 2000.

[41] Gang Zhou and Yoshiyuki Kawazoe. Localized valence states characteristics and work function of single-walled carbon nanotubes: A first-principles study. Physical Review $B, 65(15): 155422,2002$.

[42] Changwook Kim, Bongsoo Kim, Seung Mi Lee, Chulsu Jo, and Young Hee Lee. Electronic structures of capped carbon nanotubes under electric fields. Physical Review $B, 65(16): 165418,2002$.

[43] Gang Zhou, Wenhui Duan, and Binglin Gu. Electronic structure and field-emission characteristics of open-ended single-walled carbon nanotubes. Physical Review Letters, 87(9):095504, 2001.

[44] Ji Luo, L. M. Peng, Z. Q. Xue, and J. L. Wu. End potential barriers of single-walled carbon nanotubes and their role in field emission. Physical Review B, 66(15):155407, 2002 .

[45] P. von Allmen, L. R. C. Fonseca, and R. Ramprasad. Calculating the field emission current from a carbon nanotube. Physica Status Solidi (B), Basic Research, 226(1):107-113, 2001.

[46] H. Shimoyama, H. Murata, and T. Ohye. Computer simulation of electric field analysis for vertically aligned carbon nanotubes (2): electric field on the nanotube apex. Proc. SPIE, 4510:163-171, 2001. 


\subsection{Bibliography}

[47] Yung-Chiang Lan, Chun-Tao Lee, Yuan Hu, Shih-Hung Chen, Cheng-Chung Lee, Bing-Yue Tsui, and Tsang-Lang Lin. Simulation study of carbon nanotube field emission display with under-gate and planar-gate structures. Journal of Vacuum Science and Technology B: Microelectronics and Nanometer Structures, 22(3):1244-1249, 2004.

[48] A. Nojeh, B. Shan, K. Cho, and R. F. W. Pease. Ab initio modeling of the interaction of electron beams and single-walled carbon nanotubes. Physical Review Letters, 96(5):56802, 2006.

[49] Wenzhong Tang and Suresh G. Advani. Nonequilibrium molecular dynamics simulation to describe the rotation of rigid, low aspect ratio carbon nanotubes in simple shear flow. The Journal of Chemical Physics, 126(14):144711-6, 2007.

[50] R. Yabushita, K. Hata, H. Sato, and Y. Saito. Development of compact field emission scanning electron microscope equipped with multiwalled carbon nanotube bundle cathode. Journal of Vacuum Science and Technology B: Microelectronics and Nanometer Structures, 25:640, 2007.

[51] Stephanie A. Getty, Todd T. King, Rachael A. Bis, Hollis H. Jones, Federico Herrero, Bernard A. Lynch, Patrick Roman, and Paul Mahaffy. Performance of a carbon nanotube field emission electron gun. In Micro (MEMS) and Nanotechnologies for Defense and Security, volume 6556, pages 655618-12, Orlando, FL, USA, 2007. SPIE. 


\section{Chapter 3}

\section{First-Principles Study of Quantum Tunneling from Nanostructures: Current in a Single-Walled Carbon Nanotube Electron Source}

\subsection{Introduction}

Single-walled carbon nanotubes (SWNTs) are mechanically strong and physically stable structures that can be thought of as a rolled up layer of graphene. They have diameters of about a nanometer and can have lengths of up to a centimeter, resulting in very high aspect ratios. This leads to strong electric field enhancement at their tips. SWNTs are also capable of supporting current densities of up to $10^{9} \mathrm{Acm}^{-2}$, orders of magnitude higher than typical conductors such as copper and silver. These properties make them ideal candidates for electron emitters. Low turn-on voltages and brightness values of an order of magnitude higher than traditional cold field-emitters have already been demonstrated [1].

In 1928, Fowler and Nordheim proposed a model for field-electron emission (FEE) from surface emitters [2]. The model assumes that the source has a metallic density of states and, upon the application of an electric field, electrons in the metal are accelerated towards

A version of this chapter has been submitted for publication. Yaghoobi, P., Walus, K., and Nojeh, A. First-Principles Study of Quantum Tunneling from Nanostructures: Current in a SingleWalled Carbon Nanotube Electron Source. 
a one-dimensional potential barrier. If the electric field is sufficiently high, some electrons will tunnel through the junction into the vacuum. The emitted current is exponentially dependent on the barrier width and, when graphically expressed on $\ln \left[I E^{-2}\right]$ versus $E^{-1}$ scales, where $I$ is current and $E$ is electric field, will exhibit a linear behavior. This common way of expressing FEE is known as the Fowler-Nordheim plot. This model has been widely used by experimentalists to interpret the performance of electron sources, including nanotube-based emitters $[3,4]$. However, the applicability of this model to the emission process from SWNTs is not obvious and has been debated in the literature [5]. The nanotube tip does not necessarily behave like a metal; electrons are not completely free and the effect of the tip's atomic structure can be dominant. Moreover, a SWNT is a three-dimensional structure and not an infinitely-wide surface; thus, a more rigorous treatment than the Fowler-Nordheim model is required.

A SWNT emitter cannot be considered as a bulk and treated classically with macroscopic physical models. First-principles or mesoscopic models have to be used for accuracy. There has been significant work on first-principles modeling of SWNT emitters. These have shown the importance of tip orbitals in the emission process [6], the effective work function of SWNTs under a field [7], the behavior of the potential barrier [8], and the external fieldenhancement of SWNTs [9]. There has also been attempts at estimating the emission current. In particular, in their valuable work, Han et al. calculated the many body wave function of the emitting nanotube using a pseudo-potential based, time-dependent density functional theory $[10,11]$. However, in order to calculate transmission the authors applied a one-dimensional potential barrier. To the best of our knowledge, an accurate calculation of the emission current using a first-principles method, which takes into account the full three-dimentional nature of the problem and incorporates vacuum in the Hamiltonian has not been reported previously. Here, for the first time we calculate the emission current by using a three-dimentional real-space, first-principles Hamiltonian in the non-equilibrium Green's function and Fisher-Lee formulation [12]. 


\subsection{Methodology}

In a typical FEE experiment using a SWNT as an emitter, a SWNT (a few micrometers long) is grown on a cathode electrode and placed in front of an anode, such as shown in Figure 3.1(A). Our goal is to calculate the field-emission current from the SWNT using a fully first-principles approach, which is a very computationally intensive method and only allows a small section of the SWNT to be simulated. It is appropriate to choose an area close to the tip of the SWNT as it has been shown that in an external field most of the nanotube remains as an equipotential surface and the potential drop occurs mainly very close to the tip of the SWNT [8]. In other words, the emission characteristics of the device are primarily determined in the region that encapsulates the SWNT tip and vacuum, shown in Figure 3.1(B). However, such a short section of the nanotube would not recreate the strong field-enhancement that the entire nanotube would have due to its high aspect ratio. To compensate for this, we will use an electric field value that already contains the effect of the field-enhancement (several hundred times stronger than the applied value in experiments [9]).

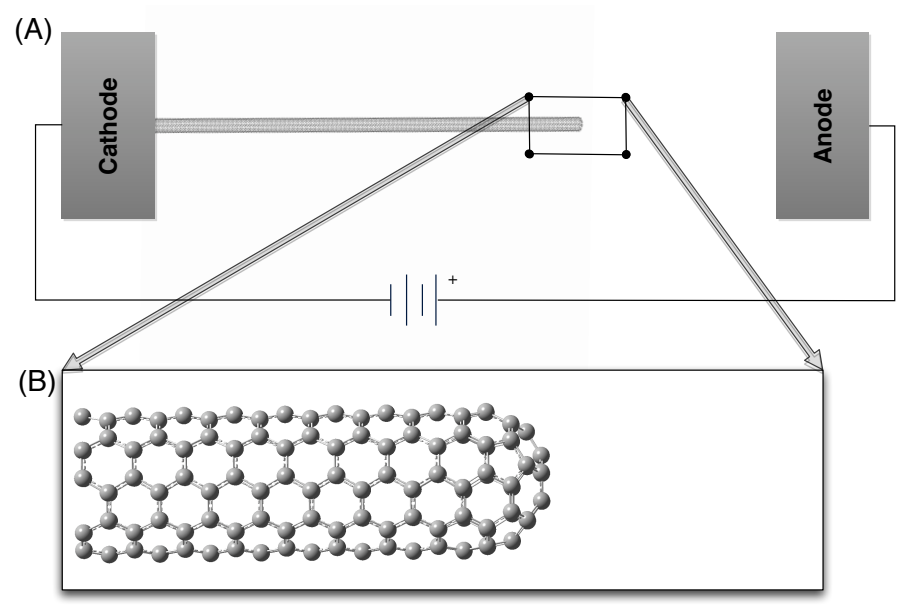

Figure 3.1: (A) Schematic representation of SWNT, consisting of the emitter grown on a cathode and biased with respect to an anode. (B) Enlarged view of the tip of the SWNT and vacuum.

We assume that this section of the nanotube tip and vacuum (shown in Figure 3.1(B) and 
referred to as the channel from now on) is connected to the continuation of the nanotube on the left side and vacuum on the right side. The current through such a channel can be calculated using the Landauer-Büttiker formula [13]

$$
I=\frac{2 q}{h} \int T(E)\left[f_{1}(E)-f_{2}(E)\right] d E
$$

where $f_{1}(E)$ and $f_{2}(E)$ are the Fermi functions of the contacts, $T$ is the transmission of the channel, $q$ is the elementary charge, and $h$ is Planck's constant.

The single particle non-equilibrium Green's function (NEGF) has the following form in the spectral representation [14]:

$$
G=\left[\left(E+i 0^{+}\right)-H_{\text {channel }}-\Sigma_{1}-\Sigma_{2}\right]^{-1}
$$

where $H_{\text {channel }}$ is the channel Hamiltonian and $\Sigma_{1}$ and $\Sigma_{2}$ are the self-energy terms that account for the coupling of the semi-infinite contacts to the channel. The transmission of the channel can be computed using the Fisher-Lee relation

$$
T=\operatorname{Tr}\left[\Gamma_{1} G \Gamma_{2} G^{\dagger}\right]
$$

where $\Gamma_{1}=i\left[\Sigma_{1}-\Sigma_{1}^{\dagger}\right]$ and $\Gamma_{2}=i\left[\Sigma_{2}-\Sigma_{2}^{\dagger}\right]$.

This formalism provides a powerful tool for calculating the transport characteristics of nano-scale structures. The proper definition of the Hamiltonian operator, $H_{\text {channel }}$, and the self-energy terms, $\Sigma_{1}$ and $\Sigma_{2}$, is essential in obtaining accurate results. Here, one challenge is how to deal with the vacuum region. Software such as TranSiesta [15] or any of the other Siesta based transport solvers are mainly designed to solve transport through atomic systems, and use atomic orbitals (such as Slater-type orbitals) as basis functions. This makes it difficult (if not impossible) to define a Hamiltonian for an FEE problem if the Hamiltonian is to encapsulates both the SWNT and vacuum, because atomic orbitals do not extend significantly into the vacuum. In our approach, however, the three-dimensional Hamiltonian is built in a real-space basis and encapsulates both the SWNT and vacuum since it is straightforward to define vacuum in a real-space basis. This is done by discretizing the kinetic energy term using difference operators in Cartesian coordinates and projecting the potential term onto the real-space basis from some other first-principles calculation 
(the potential in the area close to atoms can be calculated using available first-principles packages that employ atomic basis sets. The potential in the vacuum region far away from the atoms is determined by the applied field). By encapsulating both the SWNT and vacuum as the channel, one can easily apply the Fisher-Lee formulation in order to calculate transmission.

In order to build this Hamiltonian in real-space basis one can use the first-principles software package Gaussian 03 [16], which provides the advantage of different levels of theory and a built in utility that can project the self-consistent field (SCF) potential onto a realspace basis. A good level of theory is the Hartree-Fock (HF) formalism, which has been shown to estimate the occupied levels accurately, which are the most relevant in our calculations [17]. Density Functional Theory (DFT) would also be a natural choice, however, the most commonly used exchange-correlation (XC) functionals, namely the local density approximation (LDA) and the generalized gradient approximation (GGA), have been shown to overestimate the potential term under an electric field [18]. Siesta [19] lacks the flexibility of different levels of theory (is limited to DFT only), even for DFT it is limited to LDA and GGA. However, Siesta offers the advantage of better scaling of computation time with the size of the system. In this work we used Gaussian 03.

The self-energy terms in the real-space basis can be calculated by using the formulation developed by Appelbaum et al. [20]. This method assumes that the contacts have equipotential surfaces, with values equal to the potential energies at the corresponding ends of the channel. Each self-energy term takes the form

$$
\Sigma=-t^{2} g
$$

where $t=\frac{-\hbar^{2}}{2 m a}$, in which $a$ is the lattice spacing in the real-space basis, $m$ is the electron mass, and $g=\frac{A+S \sqrt{A^{\prime 2}-4 t^{2} I} S^{-1}}{2 t^{2}}$, where $A=E I-H_{2 D}$ ( $E$ is energy and $I$ is the identity matrix) and $A^{\prime}=S^{-1} A S$. Here, $H_{2 D}$ is the surface Hamiltonian for the three-dimensional contacts and $S$ is the diagonalizing matrix of $A$.

Once a system is coupled to contacts, it experiences a shift in energy levels, as well as level broadening [21], and these effects can have an impact on the transport characteristics of the device. However, this effect will be minimal if a long enough section of the nanotube is included in the device Hamiltonian (that is, if the contact is far enough from the tip). It 


\subsection{Results and Discussion}

is clear that computational limitations prevent a long nanotube to be included. Whether the length used in simulations is adequate can be verified by comparing the results to experiment or by performing the simulation with various nanotube lengths and determining the length at which emission current no longer changes. Since the vacuum region is included in the Hamiltonian, the right contact should have little effect on the SWNT if there is sufficient distance between the tip and the right contact. This can be easily checked by examining the gradient of the potential in vacuum. If the gradient is higher than the applied electric field then the vacuum level is perturbed by the molecule, but if the gradient is equal to the elctric-field we know that we are far enough from the molecule. The simulated device is shown in Figure 3.2 .

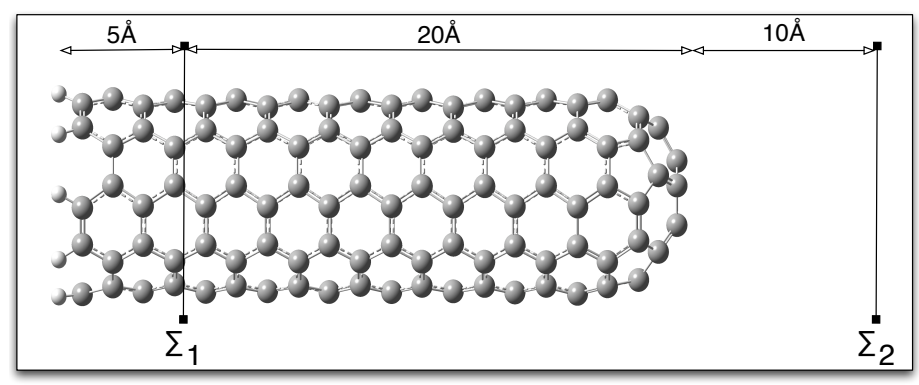

Figure 3.2: The simulated device, which encapsulates both the SWNT and vacuum. It consists of 8 unit-cells of a $(5,5)$ SWNT capped with half a $C_{60}$ molecule. The other end of the nanotube is terminated with hydrogen to avoid dangling bonds. $\Sigma_{1}$ is calculated $5 \AA$ away from the hydrogen termination because the $\mathrm{H}-\mathrm{C}$ produces an undesired dipole at the end as the result of the difference in electronegativity. $\Sigma_{2}$ is calculated $10 \AA$ away from the tip of the structure since this is where the vacuum level has been found to be unperturbed by the molecule.

\subsection{Results and Discussion}

As shown in Figure 3.2 we have simulated eight unit cells of a $(5,5)$ SWNT capped with half of a $C_{60}$ molecule. In order to speed up the geometrical optimization process in firstprinciples calculations in Gaussian 03, we first relaxed this structure in Nanohive [22], a molecular dynamics software package that uses Adaptive Intermolecular Reactive Empirical 


\subsection{Results and Discussion}

Bond Order (AIREBO) potentials [23]. These potentials are widely used in the study of carbon and hydrogen structures [24, 25]. The system was relaxed again and the electronic structure was calculated under different electric fields using the restricted HF method and the 6-31g(d) basis set in Gaussian 03. To compensate for the lack of field-enhancement in our short nanotube, a scaled external electric field (value that already contains the effect of field enhancement) was used. However, note that the actual field distribution was calculated self-consistently in the simulation and the corresponding charge accumulation is illustrated in Figure 3.3 (figure shows the Mulliken charge distribution). The hydrogen termination creates an undesired local dipole because carbon is more electronegative than hydrogen. As illustrated in Figure 3.2 that section (about two and a half unit cells) of the device was excluded in constructing the Hamiltonian.
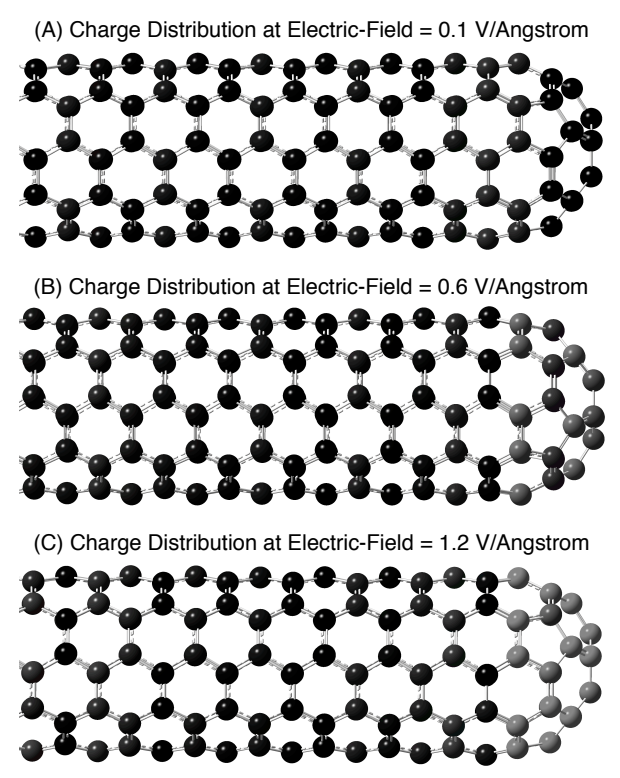

Figure 3.3: Mulliken charge distribution along the SWNT at: (A) $0.1 \mathrm{~V}^{-1}$, (B) $0.6 \mathrm{~V}^{-1}$, and $(\mathrm{C}) 1.2 \mathrm{~V}^{-1}$. The lightest grey atoms illustrate negative charges of approximately 0.17 electrons and black atoms illustrate 0 charge.

For each applied electric field and after the electronic structure calculations had converged, the SCF potential was projected onto a real-space basis with a grid spacing of $0.7 \AA$ in $\mathrm{x}$ and $\mathrm{y}$ and $0.35 \AA$ in $\mathrm{z}$ (the axis along the SWNT). At each applied electric field the left 
contact Fermi level was fixed at $4.5 \mathrm{eV}$ below vacuum level. This value was taken from the work done by Zheng et al. [8] where the authors presented an accurate calculation of the potential distribution along a micrometer long capped $(5,5)$ SWNT using a hybrid quantum/molecular mechanics method. The right contact Fermi level was fixed at the right boundary's potential level. The energy integration range was from $10 \mathrm{eV}$ above the left contact's Fermi level to $10 \mathrm{eV}$ below the right contact's Fermi level. The Fermi functions at either contacts were evaluated at room temperature. Using the aforementioned formulation, the I-V characteristic of the structure was calculated (Figure 3.4). The corresponding plot on Fowler-Nordheim scales is shown in Figure 3.5, obviously deviating from a straight line and exhibiting a non Fowler-Nordheim behavior. The current saturation behavior is very evident above $0.4 \mathrm{~V}^{-1}$, which has been previously observed experimentally among films of SWNT emitters [26, 27].

In order to better understand this saturation mechanism it is important to look at the behavior of the potential profile at the tip region of the SWNT. Figure 3.6 and Figure 3.7 illustrate the potential profile along the SWNT axis for electric fields in the psudoexponential turn-on region of the I-V curve $\left(0.2-0.4 \mathrm{~V} \AA^{-1}\right)$, and the saturation region of the I-V curve (0.4-1.2V $\left.\AA^{-1}\right)$, respectively. The dotted line represents the highest occupied molecular orbital (HOMO) level of the SWNT in each case. The tunneling current is dependent on the height and width of a potential barrier. If the height is held constant then it can be shown that the tunneling current is exponentially dependent on the width of the potential barrier [28]. In Figure 3.6 we can see that the barrier height remains almost constant and it is the width that decreases, which would explain the pseudo-exponential behavior that is seen in the $0.2-0.4 \mathrm{~V} \AA^{-1}$ region. However, as the applied field becomes stronger, the trends change, i.e. barrier width remain relatively constant (Figure 3.8), which could explain why the current remains constant (saturation regime). This behavior is very different from that of a surface emitter since as the electric field is increased for a surface emitter the barrier width would continue to decrease and maintain the exponential behavior in the IV characteristics. One way to think about this is to note that the field outside a uniformly charged, infinitely large surface is always uniform (proportional to the surface charge density, no matter how far from the surface). However, for a SWNT the field at the tip is not uniform; the sharp tip creates a nonuniform enhanced field distribution that greatly affects the potential profile of the tip and in high electric fields this local 


\subsection{Results and Discussion}

nonuniform field distribution is dominant over the external electric field in shaping the potential profile around the tip. As seen in Figure 3.7, this turns out to lead to an almost constant barrier height and width in the high-field region. We believe that this is one reason behind the observed saturation effect. This could also explain the current saturation behavior observed in other sharp emitters like nanowires [29, 30]. Also, a deep potential well exists at the tip, indicating that only a limited number of electrons can be accommodated in this well and made available for emission at any given time. These would explain the current saturation and the corresponding non Fowler-Nordheim behavior shown in Figure 3.5, which resemble the experimental plots presented by our previous experiments [31] and experiments performed by Kwo et al. [32].

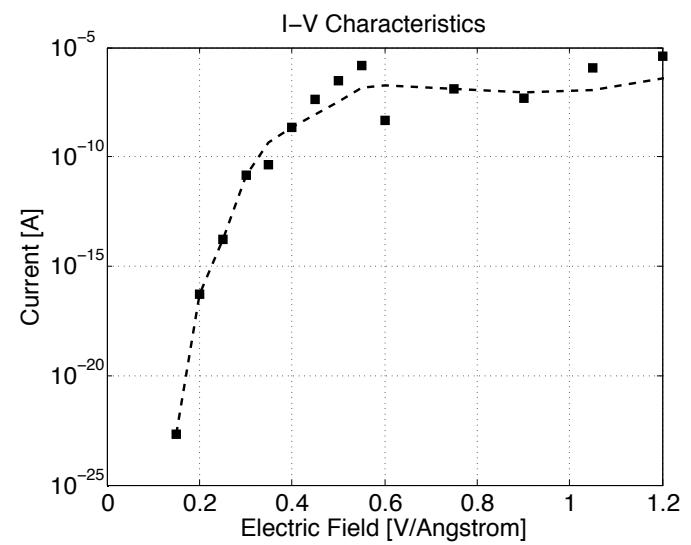

Figure 3.4: The I-V characteristics of the SWNT. The current has a pseudoexponential behavior in the turn-on $0.2-0.3 \mathrm{~V}^{-1}$ region and saturation behavior in $0.4-1.2 \mathrm{VA}^{-1}$. The dotted curve is drawn to guide the eye. 


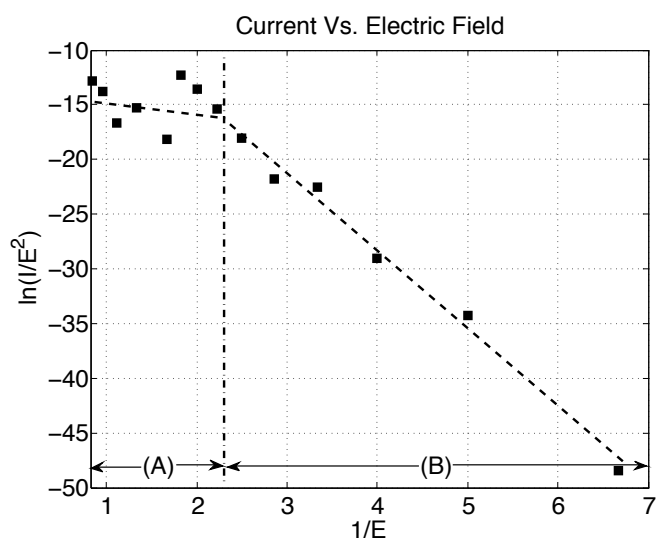

Figure 3.5: The plot of the I-V characteristics on Fowler-Nordheim scales. The fact that the graph is not a straight line, illustrated by the two fitted dotted lines (drawn to guide the eye) with different slopes, shows the non Fowler-Nordheim behavior. Section (A) is the high electric field regime and section (B) is the low electric field regime.

(A)

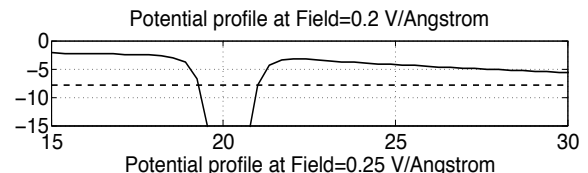

(B)

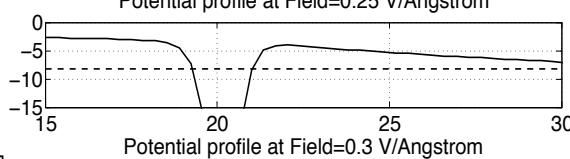

(C)

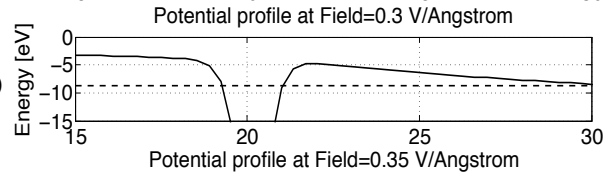

(D)

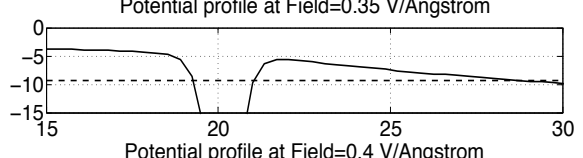

(E)

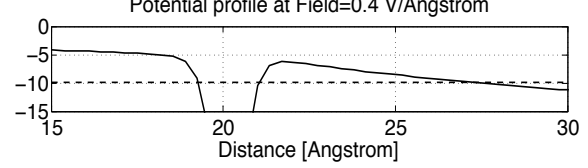

Figure 3.6: Potential profile along the the center of the SWNT in the turn-on regime. Dotted line is the HOMO level of the structure at: (A) 0.2, (B) 0.25, (C) 0.3, (D) 0.35 , and (E) $0.4 V \AA^{-1}$. 


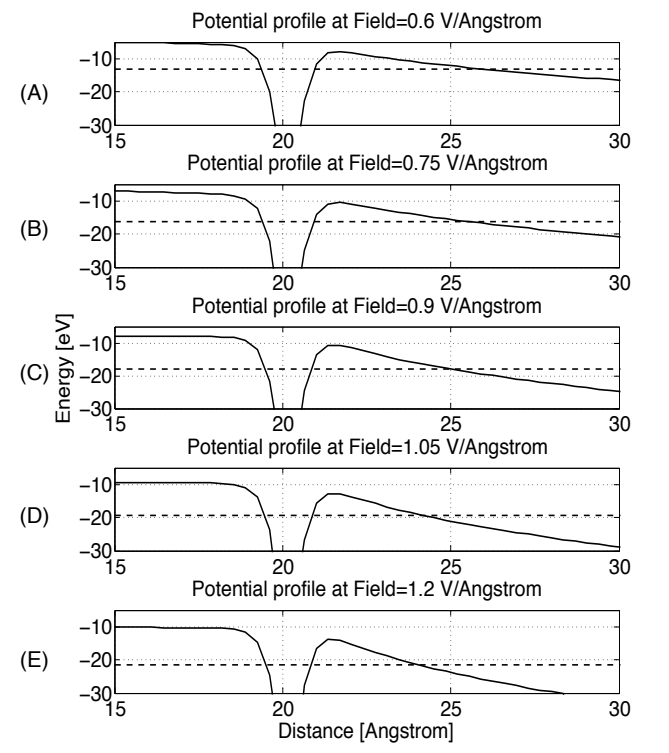

Figure 3.7: Potential profile along the the center of the SWNT in the saturation regime. Dotted line is the HOMO level of the structure at: (A) 0.6, (B) 0.75, (C) 0.9, (D) 1.05 and (E) $1.2 V \AA^{-1}$.

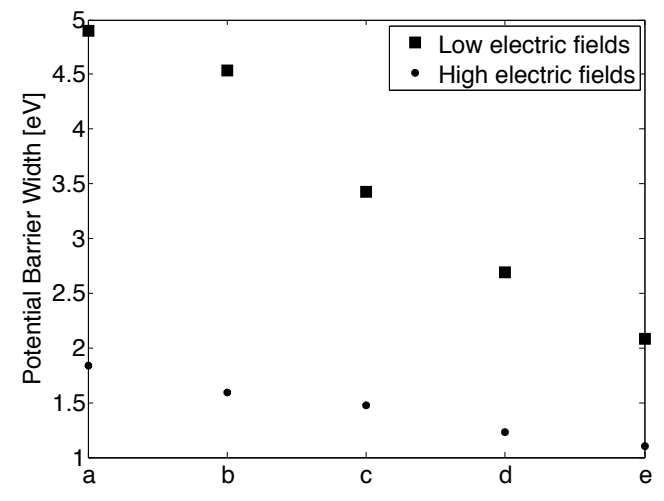

Figure 3.8: Potential barrier width between the potential well and vacuum at the HOMO energy level, circular dots represents the width at the low electric fields (corresponding to Figure 3.6) and square dots represent the width at the high electric fields (corresponding to Figure 3.7).

In many experiments so far, these deviations from the Fowler-Nordheim behavior have not been taken into serious consideration and have been overlooked by fitting a straight line into 
experimental results and presuming that carbon nanotubes do follow a Fowler-Nordheim behavior. However, our simulations suggest that SWNTs do not obey the Fowler-Nordheim behavior, which has also been suggested by Ref. [33].

Finally, we would like to emphasize that electronic transport is a non-equilibrium problem. Therefore, the Hamiltonian has to be constructed under non-equilibrium conditions. In the present work, although the Hamiltonian was constructed using the SCF method and coupled to contacts to account for shift in energy and level broadening, the transport NEGF part was not solved self-consistently. Also, due to first-principles computational limitations, only a short section of the SWNT was simulated (although the effect of field enhancement was considered by scaling the applied field and the actual potential distribution was calculated). Future work will include solving the NEGF self-consistetnly on a longer SWNT. We will also study the effect of various tip structures and adsorbents on emission current.

\subsection{Conclusion}

A first-principles approach was applied to calculating current in a SWNT field-emitter by encapsulating vacuum as part of the Hamiltonian. The transmission was calculated using a real-space basis and taking into account the full 3-D nature of the problem. The results clearly demonstrate the commonly observed current saturation behavior in electron fieldemission experiments. By further investigating the potential profile close to the tip of the SWNT, it was suggested that this saturation mechanism arises because of two main reasons. First, there exists a deep potential well that limits the number of electrons that can occupy the tip, and second and more importantly, the dependence of the emission potential barrier on applied field is significantly less in the high-field saturation regime as compared to the pseudo-exponential regime due to the dominant local electric field distribution. 


\section{Bibliography}

[1] Niels de Jonge, Myriam Allioux, Jim T. Oostveen, Kenneth B. K. Teo, and William I. Milne. Optical performance of carbon-nanotube electron sources. Physical Review Letters, 94(18):186807, 2005.

[2] R. H. Fowler and L. Nordheim. Electron emission in intense electric fields. Proceedings of the Royal Society of London. Series A, Containing Papers of a Mathematical and Physical Character (1905-1934), 119(781):173-181, 1928.

[3] Zhi Xu, X. D. Bai, E. G. Wang, and Zhong L. Wang. Field emission of individual carbon nanotube with in situ tip image and real work function. Applied Physics Letters, 87(16):163106-3, 2005.

[4] Jean-Marc Bonard, Jean-Paul Salvetat, Thomas Stockli, Walt A. de Heer, Laszlo Forro, and Andre Chatelain. Field emission from single-wall carbon nanotube films. Applied Physics Letters, 73(7):918-920, 1998.

[5] P. Yaghoobi and A. Nojeh. Electron emission from carbon nanotubes. Modern Physics Letters B, 21(27):1807-1830, 2007.

[6] Gang Zhou and Yoshiyuki Kawazoe. Localized valence states characteristics and work function of single-walled carbon nanotubes: A first-principles study. Physical Review $B, 65(15): 155422,2002$.

[7] Changwook Kim, Bongsoo Kim, Seung Mi Lee, Chulsu Jo, and Young Hee Lee. Electronic structures of capped carbon nanotubes under electric fields. Physical Review B, 65(16):165418, 2002.

[8] Xiao Zheng, GuanHua Chen, Zhibing Li, Shaozhi Deng, and Ningsheng Xu. Quantummechanical investigation of field-emission mechanism of a micrometer-long singlewalled carbon nanotube. Physical Review Letters, 92(10):106803, 2004.

[9] Seungwu Han, M. H. Lee, and Jisoon Ihm. Dynamical simulation of field emission in nanostructures. Phys. Rev. B, 65(8):085405, 2002. 
[10] Seungwu Han, M. H. Lee, and Jisoon Ihm. Dynamical simulation of field emission in nanostructures. Phys. Rev. B, 65(8):085405, Jan 2002.

[11] Seungwu Han and Jisoon Ihm. First-principles study of field emission of carbon nanotubes. Phys. Rev. B, 66(24):241402, Dec 2002.

[12] Daniel S. Fisher and Patrick A. Lee. Relation between conductivity and transmission matrix. Physical Review B, 23(12):6851, 1981.

[13] S. Datta. Electronic Transport in Mesocospic Systems. Cambridge University Press, 1995.

[14] Yongqiang Xue, Supriyo Datta, and Mark A. Ratner. First-principles based matrix Green's function approach to molecular electronic devices: general formalism. Chemical Physics, 281(2-3):151-170, 2002.

[15] Mads Brandbyge, José-Luis Mozos, Pablo Ordejón, Jeremy Taylor, and Kurt Stokbro. Density-functional method for nonequilibrium electron transport. Physical Review B, 65(16):165401, 2002.

[16] M. J. Frisch, G. W. Trucks, H. B. Schlegel, G. E. Scuseria, M. A. Robb, J. R. Cheeseman, J. A. Montgomery, Jr., T. Vreven, K. N. Kudin, J. C. Burant, J. M. Millam, S. S. Iyengar, J. Tomasi, V. Barone, B. Mennucci, M. Cossi, G. Scalmani, N. Rega, G. A. Petersson, H. Nakatsuji, M. Hada, M. Ehara, K. Toyota, R. Fukuda, J. Hasegawa, M. Ishida, T. Nakajima, Y. Honda, O. Kitao, H. Nakai, M. Klene, X. Li, J. E. Knox, H. P. Hratchian, J. B. Cross, V. Bakken, C. Adamo, J. Jaramillo, R. Gomperts, R. E. Stratmann, O. Yazyev, A. J. Austin, R. Cammi, C. Pomelli, J. W. Ochterski, P. Y. Ayala, K. Morokuma, G. A. Voth, P. Salvador, J. J. Dannenberg, V. G. Zakrzewski, S. Dapprich, A. D. Daniels, M. C. Strain, O. Farkas, D. K. Malick, A. D. Rabuck, K. Raghavachari, J. B. Foresman, J. V. Ortiz, Q. Cui, A. G. Baboul, S. Clifford, J. Cioslowski, B. B. Stefanov, G. Liu, A. Liashenko, P. Piskorz, I. Komaromi, R. L. Martin, D. J. Fox, T. Keith, M. A. Al-Laham, C. Y. Peng, A. Nanayakkara, M. Challacombe, P. M. W. Gill, B. Johnson, W. Chen, M. W. Wong, C. Gonzalez, and J. A. Pople. Gaussian 03, Revision D.01. Gaussian, Inc., Wallingford, CT, 2004. 
[17] A. S. Barnard, S. P. Russo, and I. K. Snook. Comparative hartree-fock and densityfunctional theory study of cubic and hexagonal diamond. Philosophical Magazine Part $B, 82: 1767,2002$.

[18] S. J. A. van Gisbergen, P. R. T. Schipper, O. V. Gritsenko, E. J. Baerends, J. G. Snijders, B. Champagne, and B. Kirtman. Electric field dependence of the exchangecorrelation potential in molecular chains. Physical Review Letters, 83(4):694, 1999.

[19] Jose M Soler, Emilio Artacho, Julian D Gale, Alberto Garcia, Javier Junquera, Pablo Ordejon, and Daniel Sanchez-Portal. The siesta method for ab initio order-n materials simulation. Journal of Physics: Condensed Matter, 14(11):2745-2779, 2002.

[20] Ian Appelbaum, Tairan Wang, J. D. Joannopoulos, and V. Narayanamurti. Ballistic hot-electron transport in nanoscale semiconductor heterostructures: Exact selfenergy of a three-dimensional periodic tight-binding hamiltonian. Physical Review B, 69(16):165301, 2004.

[21] Supriyo Datta. Nanoscale device modeling: the green's function method. Superlattices and Microstructures, 28(4):253-278, 2000.

[22] Brian Helfrich. Nanohive-1, Revision 1.2.0-b1. Nanorex, Inc.

[23] Steven J. Stuart, Alan B. Tutein, and Judith A. Harrison. A reactive potential for hydrocarbons with intermolecular interactions. The Journal of Chemical Physics, 112(14):6472-6486, 2000.

[24] J Marian, L A Zepeda-Ruiz, G H Gilmer, E M Bringa, and T Rognlien. Simulations of carbon sputtering in amorphous hydrogenated samples. Physica Scripta, T124:65-69, 2006 .

[25] Michael D. Furnish, Naresh N. Thadhani, and Yasuyuki Horie, editors. Molecular Dynamics Modeling of Impact-Induced Shock Waves in Hydrocarbons, volume 620. AIP, 2002.

[26] Xueping $\mathrm{Xu}$ and G. R. Brandes. A method for fabricating large-area, patterned, carbon nanotube field emitters. Applied Physics Letters, 74(17):2549-2551, 1999. 


\subsection{Bibliography}

[27] Prakash R. Somani, Savita P. Somani, S.P. Lau, E. Flahaut, M. Tanemura, and M. Umeno. Field electron emission of double walled carbon nanotube film prepared by drop casting method. Solid-State Electronics, 51(5):788 - 792, 2007.

[28] Chakraborty Gargi, C. K. Sarkar, X. B. Lu, and J. Y. Dai. Study of the tunnelling initiated leakage current through the carbon nanotube embedded gate oxide in metal oxide semiconductor structures. Nanotechnology, (25):255401, 2008.

[29] Stephane Xavier, Stefan Matefi-Tempfli, Etienne Ferain, Stephen Purcell, Shama Enouz-Vedrenne, Laurent Gangloff, Eric Minoux, Ludovic Hudanski, Pascal Vincent, Jean-Philippe Schnell, Jean-Philippe Schnell, Luc Piraux, and Pierre Legagneux. Stable field emission from arrays of vertically aligned free-standing metallic nanowires. Nanotechnology, 19(21):215601, 2008.

[30] Raghunandan Seelaboyina, Jun Huang, Jucheol Park, Dong Hun Kang, and Won Bong Choi. Multistage field enhancement of tungsten oxide nanowires and its field emission in various vacuum conditions. Nanotechnology, 17:48404844, 2006.

[31] A. Nojeh and R. F. Pease. Field-electron emission from single-walled carbon nanotubes lying on a surface. In Canadian Conference on Electrical and Computer Engineering, Vancouver, British Columbia, Canada, 2007.

[32] J. L. Kwo, Meiso Yokoyama, W. C. Wang, F. Y. Chuang, and I. N. Lin. Characteristics of flat panel display using carbon nanotubes as electron emitters. Diamond and Related Materials, 9(3-6):1270 - 1274, 2000.

[33] Gang Zhou and Yoshiyuki Kawazoe. Localized valence states characteristics and work function of single-walled carbon nanotubes: A first-principles study. Phys. Rev. B, 65(15):155422, 2002. 


\section{Chapter 4}

\section{Summary, Conclusions and Further Work}

Electron sources, which are the fundamental components of many important devices and equipments, ranging from vacuum tubes to electron microscopes, continue to be the performance bottle neck in many applications. By investigating the physical properties of novel materials that could enable better electron sources, we may indeed significantly improve many existing electron-beam systems and even make new application possible, making electron sources more relevant to our daily lives.

As introduced in chapter two, carbon nanotubes (CNTs) are one such material that, over the past two decades, have shown to be a strong candidate for modern electron sources. The electrical and mechanical properties of CNTs have attracted researchers in many different disciplines to use them in various applications. CNT-based electron sources are just one example. Chapter 2 provided a review of CNT electron sources in the existing literature. The common understanding of their electron emission mechanisms in CNTs and the discrepancy between theoretical and experimental results were discussed. Most notably is the current emission saturation behavior in field electron-emission from CNTs, which deviates significantly from the prediction of the well accepted model proposed by Fowler and Nordheim for surface metallic emitters.

Chapter 2 also discussed the most recent theoretical works on carbon nanotube based electron sources and pointed out the challenges that exist in modeling these devices using first-principles methods and, as a result, the lack of a thorough understanding of carbon nanotube electron emitters. Although several valuable first-principles simulation works have been done on nanotube electron emitters, they have predominantly focused on the 
investigation of the electronic structure at the nanotube tip. A very limited number of works study the transport problem during emission. To the best of the author's knowledge, the work presented in chapter 3 is the first complete treatment of the problem, using a threedimensional, first-principles calculation to predict the emission current and quantitatively explain the saturation mechanism.

The methodology described in this paper also forms the basis of a new quantum-mechanical transport solver program, which is very flexible and can handle vacuum as part of the structure. Although in this particular instance it was used for nanotube electron emitters, this method can be applied to a wide variety of other problems that involve a vacuum region. Examples include scanning tunneling microscopy and tunnel junction devices. Current commercial first-principles transport solvers (such as TranSiesta or QuantumWise/Aomistix) cannot handle these problems due to being limited to atomic basis sets (which do not extend into vacuum).

This methodology can also be used to study field-electron emission from CNTs in greater detail. As discussed in chapter 2, different researchers have looked into adsorbents as playing an important role in the emission saturation behavior. At the same time adsorbents like cesium have shown to decrease the work function of CNTs by $2.4 \mathrm{eV}$ [1]. These experimental findings can be studied theoretically using the methodology presented in chapter 3 .

Of course, investigating different CNT chiralities and their effect in field-electron emission using this method is an obvious immediate work that can easily be performed. Also, electron stimulated field-emission, again discussed in chapter 2, can be pursued further theoretically by calculating the gain of this device.

Appendix A presents the required equipments and initial experiments for characterizing electron emission from forests of CNTs or a single CNT. For example, in the ultra high vacuum chamber using the nano manipulator a single CNT can be isolated and fieldelectron emission experiments can be performed. Also to better understand the capabilities of CNT electron sources, different shapes and forms of CNT forests can be grown to test the limits of CNT based electron sources. 


\section{Bibliography}

[1] S. Suzuki, C. Bower, Y. Watanabe, O. Zhou. Work functions and valence band states of pristine and cs-intercalated single-walled carbon nanotube bundles. Applied Physics Letters, 76:4007, 2000. 


\section{Appendix A}

\section{Experimental Setup}

In order to study nanotube electron sources experimentally, I have been involved in building these systems: I built a chemical vapor deposition (CVD) reactor and finalized two different recipes to grow carbon nanotubes. To verify the growth results and have access to a high vacuum chamber, I reassembled an old Philips 505M scanning electron microscope back to working condition. I also helped with designing and assembling an ultra high vacuum chamber, which is an ideal environment for characterizing electron emission from carbon nanotubes and, in particular, their emission saturation behavior.

\section{A.1 Chemical Vapor Deposition system}

The CVD setup is shown in Figure A.1. It consists of a quartz tube that is heated with a tube furnace. There are four available lines for gases (ethylene, methane, hydrogen, and argon) that can be mixed together through a manifold (Figure A.2). The flow of each gas can be controlled accurately $( \pm 0.5-1.5 \mathrm{sccm}$ for ethylene and $\pm 10-20 \mathrm{sccm}$ for methane, hydrogen, and argon) using a thermal-based mass flow controller. Different combinations of these gases can provide different recipes for growing carbon nanotubes. 


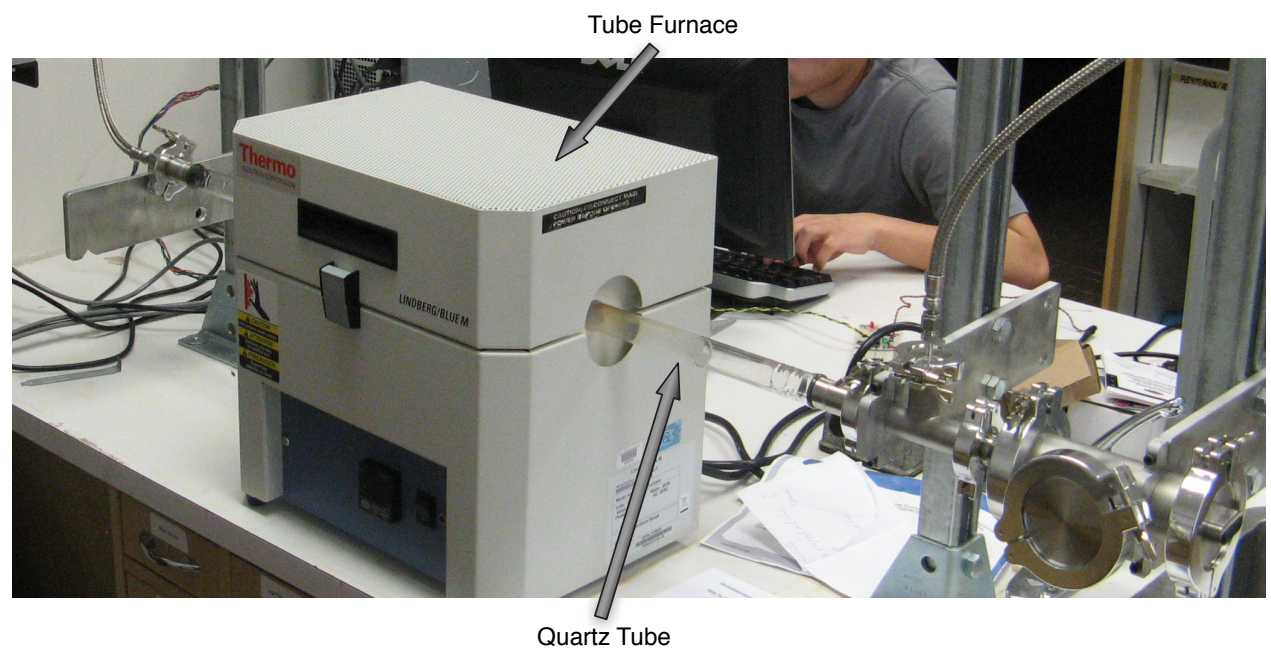

Figure A.1: CVD setup consisting of a tube furnace and a quartz tube.

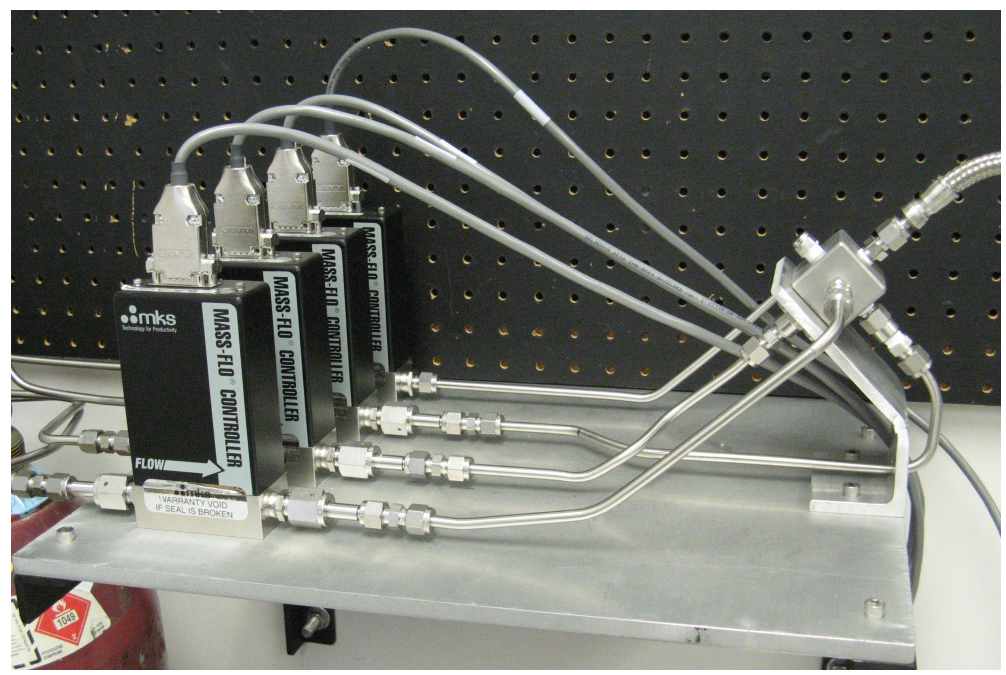

Figure A.2: The manifold setup where precise flows of the gases are mixed.

The first recipe was developed for growing CNTs lying on a surface based on the method of Kong et al. [1]. This recipe required preparation of a catalyst solution, which contains iron, alumina, and molybdenum nano particles dissolved in methanol. Devices are prepared by first evaporating molybdenum contacts on a silicon wafer, which is coated with a 1 um 
layer of silicon oxide. Then patterning PMMA resist to deposit the catalyst solution where carbon nanotubes will grow from.

A typical growth using this recipe would involve heating up the sample to $900^{\circ} \mathrm{C}$ while flowing hydrogen (2000 sccm). Once the temperature has stabilized, I would start the flow of ethylene $(20 \mathrm{sccm})$, methane $(500 \mathrm{sccm})$, and reduce the flow of hydrogen to 500 sccm for 10 to 15 minutes. A few SEM images of these growths are shown in Figure A.3. This recipe can be used for many different kinds of devices, such as those needed for field electron-emission from CNTs that are lying on a surface [2] and electron stimulated field-emission [3].

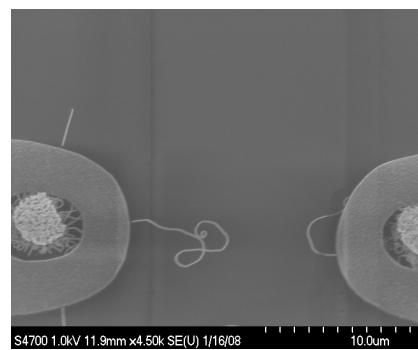

(A)

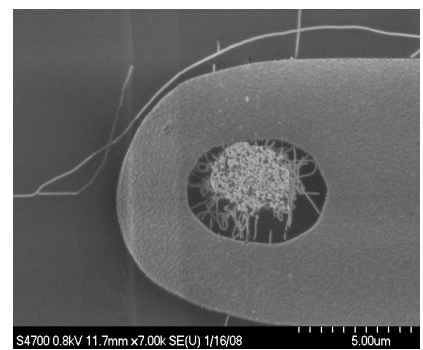

(B)

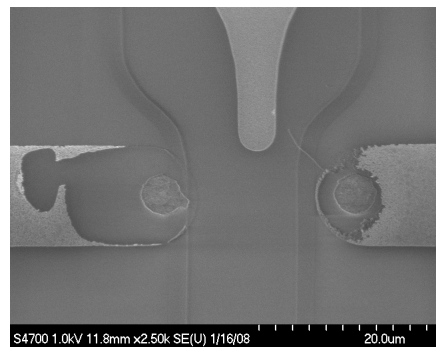

(C)

Figure A.3: SEM images using a FE Hitachi S4700 SEM of: (A) CNTs grown from catalyst islands (B) Zoomed in image of the catalyst islands and CNTs (C) A long CNT grown from a catalyst island towards an electrode.

The second recipe has been developed for growing millimeter long forests of CNTs. It is a mixture of the recipes proposed by Hata et a.l [4], Zhang et al. [5], and Chakrabarti et al. [6]. Molybdenum contacts were evaporated on a silicon wafer coated with 1um layer of silicon oxide, then $10 \mathrm{~nm}$ of alumina and 1-2nm of iron were evaporated respectively as catalysts for growing forests of carbon nanotubes. A typical growth would entail heating the sample up to $750^{\circ} \mathrm{C}$ while flowing hydrogen $(500 \mathrm{sccm})$ and argon $(200 \mathrm{sccm})$ until the temperature has stabilized and then start the flow of ethylene (20 sccm) and reducing argon and hydrogen to $120 \mathrm{sccm}$ and $80 \mathrm{sccm}$ respectively for 30 minutes. SEM picture of these forests on patterned electrodes are shown in Figure A.4. To identify whether this recipe grows MWNTs or SWNTs TEM images of these nanotubes were taken (Figure A.4(C)). The fringes on each side of the nanotube show more than one layer of carbon and therefore confirms that this recipe grows forests of MWNTs. 


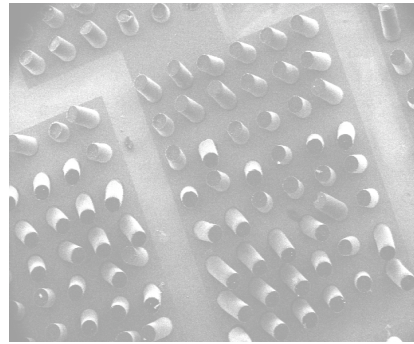

(A)

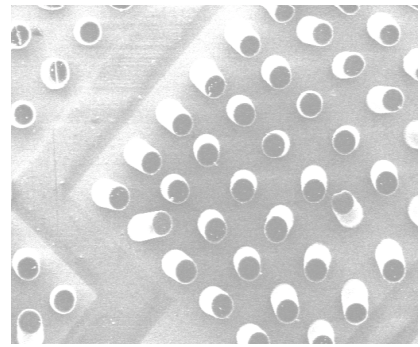

(B)

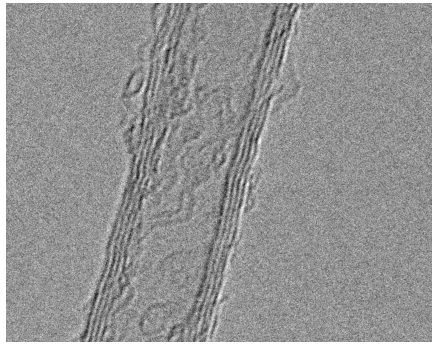

(C)

Figure A.4: SEM images using a Philips 505M SEM of: (A) CNT forests grown from patterned electrode (field of view of $2 \mathrm{~mm}$ ) (B) Zoomed in image of CNT forests grown from patterned electrode (field of view of $1.5 \mathrm{~mm}$ ) (C) TEM image of the grown MWNT using an FEI Titan TEM.

\section{A.2 Scanning Electron Microscope}

A Philips 505M scanning electron microscope (SEM) was donated to our laboratory by Stanford University (Figure A.5) . It had been fully disassembled for the move and delivered in many different pieces. Over a few months I reassembled all the pieces back together and tested for its operation section by section. One of the big challenges to get the system working initially was to reach vacuum so that I could turn on the electron beam and test the optics. Also this SEM was built long before modern computerized image capturing devices, however, it had been updated with one. Making this part of the SEM work proved to be one of the most challenging tasks in bringing the SEM back to its working condition. This device can capture high resolution images. A few examples of such images are shown in Figure A.6. 


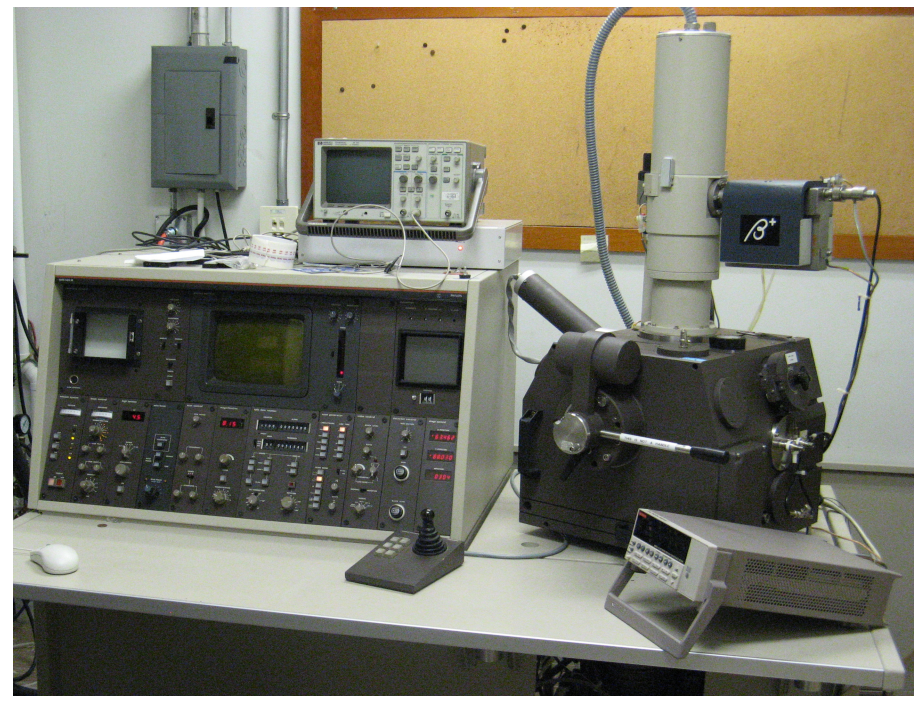

Figure A.5: Reassembled and working Philips 505M SEM.

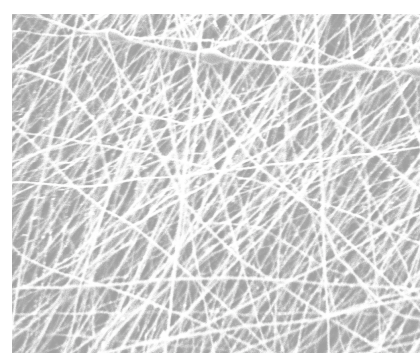

(A)

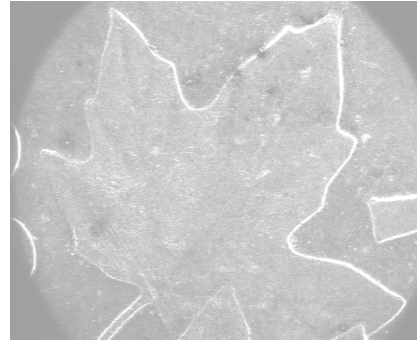

(B)

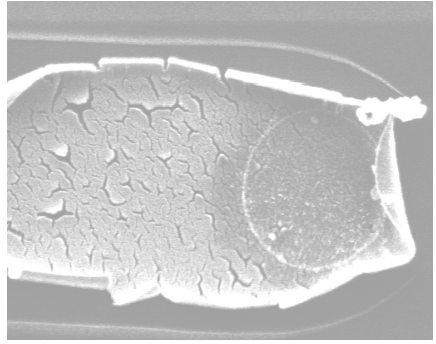

(C)

Figure A.6: SEM images using a Philips 505M SEM of: (A) Carbon fibers (B) A canadian penny $(\mathrm{C})$ a pealed electrode.

This SEM not only provides a very convenient way of inspecting different nanotube growth recipes, it also provides a high vacuum chamber that can be used for characterizing electron emission from CNTs and has been used for field-emission and stimulated field emission experiments. 


\section{A.3 Ultra High Vacuum Chamber}

An ultra high vacuum (UHV) chamber can provide pressures as low as $10^{-12}$ Torr depending on the cleanliness of the chamber. This level of pressure translates into a mean free path of 100, $000 \mathrm{~km}$ for gas molecules. This is an ideal environment for characterizing CNT electron emitters because it would tremendously slow down the deposition rate of nitrogen or other atoms or molecules that could greatly affect the electronic structure of CNTs and therefore their electron emission behavior. For example, as discussed in chapter 2, adsorbents could play a very important role in the current saturation behavior of CNTs [7] and by running experiments in an ultra high vacuum environment, one can significantly slow down adsorption on CNTs and obtain an accurate picture of electron emission behavior. Figure A.7 is a picture of our UHV chamber, which I have helped design and assemble. We also have a high precision XYZ mechanical stage with sub-micron precision and a transfer arm, which is currently being delivered. We also have a nano-manipulator, which is going to be mounted on this stage. This setup would give us sub-nanometer manipulation capabilities, which is ideal for manipulating single nanotubes.

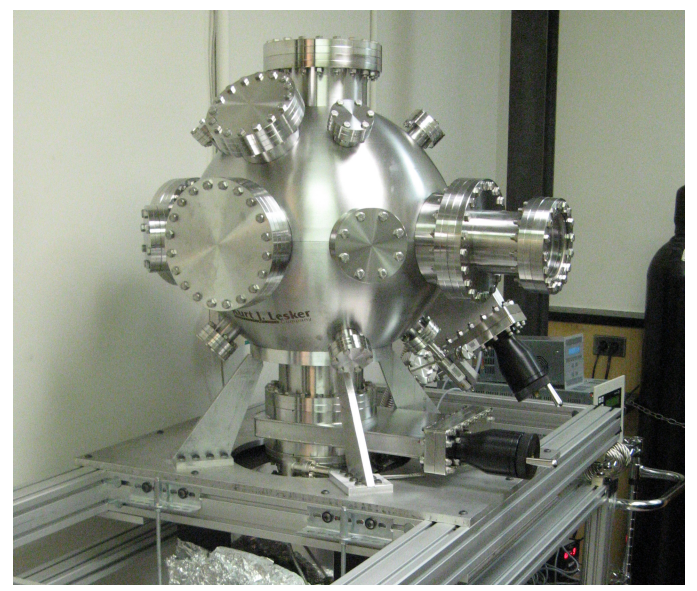

Figure A.7: Assembled and working UHV chamber. 


\section{A.3. Bibliography}

\section{Bibliography}

[1] Jing Kong, Hyongsok T. Soh, Alan M. Cassell, Calvin F. Quate, and Hongjie Dai. Synthesis of individual single-walled carbon nanotubes on patterned silicon wafers. Nature, 395(6705):878-881, 1998.

[2] A. Nojeh and R. F. Pease. Field-electron emission from single-walled carbon nanotubes lying on a surface. In Canadian Conference on Electrical and Computer Engineering, Vancouver, British Columbia, Canada, 2007.

[3] A. Nojeh, W. K. Wong, E. Yieh, R. F. Pease, and H. Dai. Electron beam stimulated field-emission from single-walled carbon nanotubes. Journal of vacuum science and technology. B. Microelectronics and nanometer structures. Processing, measurement and phenomena, 22(6):3124-3127, 2004.

[4] Kenji Hata, Don N. Futaba, Kohei Mizuno, Tatsunori Namai, Motoo Yumura, and Sumio Iijima. Water-Assisted Highly Efficient Synthesis of Impurity-Free Single-Walled Carbon Nanotubes. Science, 306(5700):1362-1364, 2004.

[5] C. Zhang, S. Pisana, C.T. Wirth, A. Parvez, C. Ducati, S. Hofmann, and J. Robertson. Growth of aligned millimeter-long carbon nanotube by chemical vapor deposition. Diamond and Related Materials, 17(7-10):1447 - 1451, 2008.

[6] Supriya Chakrabarti, Takeshi Nagasaka, Yuya Yoshikawa, Lujun Pan, and Yoshikazu Nakayama. Growth of super long aligned brush-like carbon nanotubes. Japanese Journal of Applied Physics, 45(28):L720-L722, 2006.

[7] Kenneth A. Dean and Babu R. Chalamala. Current saturation mechanisms in carbon nanotube field emitters. Applied Physics Letters, 76(3):375-377, 2000. 